ZASPiL Nr. 36 - December 2004

Blocking Resultative Secondary

Predication in Russian

Anatoli Strigin 


\title{
Blocking resultative secondary predication in Russian
}

\author{
Anatoli Strigin \\ ZAS Berlin
}

\begin{abstract}
The paper explains the absence of resultative secondary predication in Russian as arising from a conflict of inferential interpretations. It formalises the framework necessary to express this proposal in terms of abductive reasoning with Poole systems in Gricean contexts. The conflict is shown to arise for default rules regulating alternative realisation of verb-internally specified consequent states. The paper thus indicates that typological variation may be due not only to different parameter values but to general inferential properties of the syntaxsemantics mapping. The proposed theory also contradicts some widespread proposals that the absence of resultative secondary predication is due to the absence of some particular language feature.
\end{abstract}

\section{Introduction}

\subsection{The problem of resultatives in Russian}

The three men in a boat whose tale was told by Jerome K. Jerome were trying one day to open a tin of pineapple with a boat mast for lack of better opening implements. Here is how the story went:

We beat it out flat; we beat it back square; we battered it into every form known to geometry - but we could not make a hole in it.

The point of this story in the present context is that to tell it in Russian you have to use paraphrases for the construction exemplified by beat it out flat and beat it back square. Russian does not have this kind of construction, termed resultative construction (or RC, for short), although it has words which closely correspond to the lexical constituents: bit' for beat, ploskij for flat orkvadratnyj forsquare. A Russian translation of the passage could look as follows: 
My raspl'uščili etu banku v lepešku, potom sdelali eë snova kvadratnoj; my pridavali jej samyje udivitel'nyje geometričeskije formy, no probit' otverstija ne smogli. (Jerome K. Jerome, Three men in a boat, Moscow, Raduga, 2003, a bilinguial edition, Russian translation by E. Lineckaja)

It is instructive to consider a backwards translation of the Russian passage into English to see how good Russian paraphrases the construction. A translation could look like this:

We flattened this tin into a flat cake, then we made it again square; we imparted most wonderful geometrical forms to it, but we were not able to beat-through a hole.

The two prefixes set in boldface in the Russian text should be briefly described here, though they will occupy us later. The prefix ras- is a more or less pure perfectivizing prefix in this context, since it helps to assert that the tin was indeed made flat; pro- is also a perfectivizer but in addition it specifies that the beating results in penetrating some surface. This additional meaning is indicated in the backward translation by the add-on -through. The clumsy clause we could not beat-through a hole should show that the Russian verb probit', beat through, allows to drop the argument which specifies the location of the hole.

In sum, Russian does not have the resultative construction, but it seems to be able to express its sense rather closely. The subject of this paper is the question why Russian has no such resultative construction to express the desired sense.

The question might have a simple answer: the absence of this construction in Russian is a purely accidental feature. But, if by pure accident some mechanism or pattern of interpretation present in English and German are absent in Russian or French, which has no such construction either, these particular mechanisms of interpretation must be assumed to be unrelated to other properties of languages.

The paper in contrast takes a closer look at the hypothesis that the resultative interpretation of a syntactic structure which could be similar to that of English is blocked in Russian by an interaction of the general mechanisms of interpretation with some particular properties of Russian. 
It is thus at variance with the prevailing tendency to explain the absence of resultative secondary predication in a language by reference to some absent feature of the language. Examples of such theories are Legendre (1997), who claims that French lacks some syntactic feature, and Wunderlich (1997), who proposes that languages without RC lack a lexical operation.

The property which differentiates between English and Russian is the grammaticalization of the cognitive operation of event bounding in Russian. English does not grammaticalize this operation. Grammaticalization here means that the operation is obligatorily explicitly manifest in Russian perfective verbs. The English perfective verb forms grammaticalize a different though rather similar operation of constructing a perfective state.

Resultative construction shares part of its semantics with event bounding. This similarity is crucial in determining its absence in Russian.

We will assume an inferential theory of meaning in Gricean contexts. This theory uses reasoning in a context both to provide an interpretation of syntactic structures and to generate them starting from the communicative sense. The important property of Gricean contexts is the organisation of inferential resources which reflects both the speaker's and the hearer's perspectives on the recoverability of the communicative sense.

One major principle of resource structuring is management of alternative interpretations. The general mechanism employed by the proposed theory to manage alternatives is the use of negation as failure. We will model the inferential theory of meaning and negation as failure using abductive frameworks. Negation as failure interacts with the grammaticalized event bounding operation and blocks the resultative secondary interpretation in Russian, since the latter is partly an alternative. This interaction hypothesis will be called RSP blocking hypothesis. RSP stands for resultative secondary predication. The term was chosen because the resultative structures exemplified in J. K. Jerome's story are usually considered in linguistics to be instances of a more widespread phenomenon called secondary predication. Russian has some uses of secondary predication which are exemplified by English or German ${ }^{1}$, but it also seems to have a lot more uses which are not observed in these two languages. The paper therefore

\footnotetext{
${ }^{1}$ In particular, Russian exhibits depictive secondary predication, cf. (1)
} 
is limited to investigating the RSP blocking hypothesis only. A series of papers by Assinja Demjjanow and Anatoli Strigin (Demjjanow and Strigin, 2003, 2001; Strigin and Demjjanow, 2001) give a broader picture of secondary predication in Russian.

Note that the RSP blocking hypothesis only asserts that Russian as it is cannot accommodate RSP. It does not make any statements as to why Russian has developed into the language it is.

The plan of the paper is as follows. We will first review the basics of the resultative construction in English in some detail as well as a proposal by Levin and Rappaport Hovav which suggests why the RSP blocking hypothesis could be of interest in explaining the absence of $\mathrm{RC}$ in Russian. These motivating remarks as a starting point, some interesting proposals of a formal semantics of $\mathrm{RC}$ and in general on the role of states in the semantics of verbs will be reviewed. It will become clear that these analyses need an amendment to adequately reflect constraints on $\mathrm{RC}$. In the course of making these amendments a distinction between bounding events and perfective events will be formulated, which will play a crucial role in the RSP blocking hypothesis. We will furthermore sketch a theory which can integrate the proposal by Levin and Rappaport Hovav and the improved semantics. The theory is based on the inferential mechanism of abduction. A formalisation of the theory in terms of the so-called Poole systems will be suggested and a treatment of Russian and the absence of $\mathrm{RC}$ in it will be proposed in this formalised framework. A separate discussion of prepositional resultatives and some conclusions come last.

\subsection{The resultative construction (RC) in English}

There is a vast amount of literature on RSP in English and on secondary predication which cannot be reviewed here. Two very informative recent books on this subject which can be consulted for a review of the relevant literature, are Winkler (1997) andRothstein (2001). The description below follows Levin and Rappaport Hovav (1995). Levin and Rappaport Hovav

(1) On pjet čaj gor'ačim

He-nom drinks tea-acc hot-instr

He drinks his tea hot

Here -nom -acc and -instr indicate the nominative, accusative and instrumental cases 
(1995) introduce the phenomenon in the following way:

A resultative phrase is an XP that denotes the state achieved by the referent of the NP it is predicated of as a result of the action denoted by the verb in the resultative construction

Thus, the resultative construction (RC) consists of a verb, an NP, and an $\mathrm{XP}$, integrated semantically into an interpretation pattern. This description does not specify the syntactic relations between the three constituents, nor does it specify why the pattern result of the action is used to interpret the syntactic pattern. The construction shows some variation, as the useful typology of Levin and Rappaport Hovav shows below.

\subsubsection{A typology of RC}

The examples are mostly taken from Levin and Rappaport Hovav (1995). They are restricted to cases with $\mathrm{XP}=\mathrm{AP}$, because other cases raise other problems in Russian. Possible Russian equivalents are indicated, too.

RC based on transitive verbs If a resultative phrase occurs with a transitive verb, it may be predicated only of the object of the transitive verb, never of its subject (direct object restriction, DOR). If the phrase is predicated of the subject, it loses its resultative interpretation.

(2) ... while she soaps me slippery all over [D. Pryce-Jones, The Afternoon Sun, 186]

No exact replica of the pattern is possible as a Russian version, cf (3):

(3) * Ona mylila men'a skolzkim

He soaped/was soaping me slippery

Sche soaped me slippery

Nevertheless, a rendering of the communicative sense is possible. It would be a separate clause for the adjective which has a resultative reading, (4).

(4) Ona namylila men'a tak čto ja stal skolzkim She soaped me so that I became slippery She soaped me, so that I became slippery 
PC based on unergative verbs Unergative verbs have no object. DOR is satisfied, however, in three ways.

(a) by the addition of a fake reflexive.

(5) Dora shouted herself hoarse

The Russian literal translations is impossible, with or without a reflexive $(6)$.

(6) *Ona kričala (seb’a ) xriploj

She shouted (herself) hoarse

She shouted herself hoarse

But the version with a prepositional phrase with the meaning similar to the adjective is possible, in approximately the same sense, $(7)$.

(7) Ona kričala do xripoty

She shouted till hoarseness

She shouted/used to shout herself hoarse

(b) by adding the non-subcategorised referent NP, as if it were a direct object (pseudo-transitive verbs)

(8) ... the dog barked him awake [S. Paretsky, Blood shot, 183]

No adequate literal translation is possible here. The nearest equivalent is to say that the dog woke him up by barking.

(c) by adding the non-subcategorised referent NP which is inalienably possessed by the subject(contains a possessive pronoun referring to the subject)

(9) Sylvester cried his eyes out

A similar communicative sense can be transmitted with the help of a perfectivized prefixed verb vy-plakat', (10),

(10) Ona (sebe)

glaza vyplakala

She herself-da tive eyes cried-out

She cried her eyes out

Here, vy- is a regular prefixation indicating some sort of removal, so that not only eyes can be cried out, but anything which can be thus removed, e. g. grief, (11). 
(11) Ona svoe gore vyplakala

She her grief cried-out

She cried her grief out

It is noteworthy that no equivalent with the simplex imperfective verb exists.

RC based on unspecified object verbs Transitive verbs which allow intransitive uses with an unspecified object interpretation may allow a resultative XP with a different, non - selected object.

(12) Having ... drunk the teapot dry ... [E. Dark, Lantana Lane, 94]

No direct translation is possible here into Russian. However, a prepositional phrase with a de-adjectival adverb is possible, (13), though very strange, and do dna (to the bottom) instead of dosuxa would be more appropriate.

(13) Ona vypila čajnik dosuxa

She drank-o ut the teapot to-dry

She drank the teapot dry

$\cdot$

RC based on passive and unaccusative verbs

(14) She was shaken awake by the earthquake

(15) The gate swung shut

No literal translations are possible, but similar communicative senses may be communicated by saying that she woke up because the earthquake's tremors shook her or that the gate rotated and closed i. e. by using an explicit clause.

This assortment of Russian more or less exact communicative equivalents shows the three strategies of rendering the communicative sense of RSP by the means available in Russian: a prefixed - hence perfectivised - verb, as in (11), a prepositional phrase with often a deadjectival noun, as in (7), or a deadjectival adverb, as in (13). Where these means are not available more general means, like subordinate clauses, come into play. 
There does not seem to be any correspondence between the type of $\mathrm{RC}$ and a preferred rendering type in Russian.

\subsubsection{The structure of the mapping between the syntax and semantics of RC in English}

DOR seems to have acquired a dubious status of late. Rappaport Hovav and Levin (2001) present some material intended to show that DOR cannot be maintained and suggest a new approach to resultatives. Rappaport Hovav and Levin (2001) suggest that the interpretation of the RC involves two events which stand in a causative relation: the one described by the verb and the one described by the resultative XP. The principle (16) postulated by a number of researchers as either derived or normative regulates their realisation in the syntax.

(16) ARGUMent-PER-SUbevent CONDition There must be at least one argument XP in the syntax per subevent in the event structure

Rappaport Hovav and Levin (2001) conclude

If this condition is correct, argument realization patterns should reflect event complexity, in that event structures with two subevents must give rise to sentences with both a subject and an object, while simple event structures require only a subject.

The authors also think that

An English verb-result XP combination describes a complex event consisting of two subevents, one represented by the verb and the second by the result XP.

Levin and Rappaport Hovav agree with Wunderlich (1997) that the adequate representation level is the one of lexical semantics-syntax mappings, hence the semantics is basically that of a lexical conjunction, and also adopt the claim by Kaufmann (1995) and Wunderlich (1997) that in a lexically specified conjunction of two events the second event serves to support inferences about the first event. The causal element in the representation is then somehow inferred. For examples like (12) the inference is rather involved. Ignoring the details, the proposal seems to be that if two events are causally related at some level of linguistically relevant representation, and 
the caused event is realized via RSP, there must be an expressed argument which is the bearer of this event.

Discussing (17), Rappaport Hovav and Levin are then forced to assume that the relevant situation is formed around one event and not around two events, and therefore does not provide the second argument, to explain the observed contrast.

(17) a. Robin danced out of the room

b. ?? Robin danced herself out of the room

This move by Rappaport Hovav and Levin could seem odd, since the two events may beassumed on the basis of our intuitions: one event is that of Robin dancing and one event of Robin moving out of the room. They propose therefore the following argument, which they consider valid for $\mathrm{RC}$ in general:

- two events, the event of Robin moving and the event of her getting out of the room are coincident in time and space

- therefore, they are co-identified as one event for linguistic purposes

- this is done because the preferred expression of a situation is the one giving it the tightest event structure

- this preference follows from some version of Grice's maximum of quantity (Grice, 1975)

- the co-identification is therefore obligatory unless some other pragmatic factors override this maxim and the structure of two events is assumed for the situation.

Levin and Rappaport Hovav (1999) give some examples of such reorganised structure, cf. (18).

(18) Then, without another word, he withdrew from the kitchen and sauntered his Bermuda-shorted self through the front door. (D. M. Davidson, Killer Pancake, Bantam, New York, 1995, p.63

The question of an explanation of the absence of RSP in Russian was not raised by Levin and Rappaport Hovav. Wunderlich (1997), whose account 
may be integrated with the discussion by Levin and Rappaport Hovav, postulates an operation of lexical adjunction which creates a new argument of the verb in $\mathrm{RC}$, the resultative XP. He assumes that if $\mathrm{RC}$ is not present in a language, this must be due to the absence of the operation. But this proposal merely appeals to the unknown properties of lexical processes. As long as we have no account of the properties which disallow this lexical operation we look for more general ways of explanation or maintain that it is absent by chance. However, the discussion by Levin and Rappaport Hovav contains an interesting idea which can be explored to find a more systematic explanation for Russian facts. In the rest of the paper we shall lay out a proposal of how to do it. Let us start by taking a closer look at potential mechanisms behind the discussed proposal.

\subsubsection{Gricean contexts as a model of explanation}

The part of the proposal of Levin and Rappaport Hovav most interesting in the present context is that pragmatic constraints are claimed to dictate first the semantic patterning of the conceptual information about situation and second the mapping of the patterns to syntax. The following is a simple, but for our purposes adequate reconstruction of what happens in such contexts where pragmatic factors determine the mapping of communicative sense to syntax in at least two stages. Let us call them Gricean contexts.

We assume that both the patterning and its mapping to syntax in Gricean contexts are done by two sorts of default rules: patterning rules and linking rules. They interact in that if one rule of a set applies, it blocks some or all of its alternatives. Blocking may be cancelled under certain conditions, though. Consider these basics in more detail, to see how they can be used to develop an explanation for our main question.

Suppose we have the description of the situation which provides the communicative sense for the sentence. Consider the situation patterning where no event identification takes place. Let the patterning be given by some complex condition condition $(e 1, e 2)$. This condition is taken to adequately characterise the portion of the situation which is mapped to verb arguments. It may also be said to pattern the information in the situation. The situation in which the identification takes place is then characterised by additional conditions, which do not contradict condition $(e 1, e 2)$, but 
specify it further:

$$
\begin{aligned}
& \text { condition }(e 1, e 2) \& \text { sametime }(e 1, e 2) \& \text { sameplace }(e 1, e 2) \\
& \rightarrow \text { co_identify }(e 1, e 2)
\end{aligned}
$$

Thus, we have two kinds of patterning, with and without co-identification, of which one is a specification of the other.

The rules mapping patterned information to syntactic structures constitute a separate building block of linking rules. The predicates map $1(x, k)$ and $\operatorname{map} 2(y, j)$ in the example below stand for theories which map entities and relations of semantic representations to entities and relations of syntactic representations.

condition $(e 1, e 2) \&$ semrole $1(e 1, x) \&$ semrole $2(e 2, y) \rightarrow$ $\operatorname{map} 1(x, k) \& \operatorname{map} 2(y, j)$

condition $(e 1, e 2)$ \& co_identify $(e 1, e 2)$ \& semrole1 $(e 1, x)$ \& semrole $2(e 2, x) \& x=y \rightarrow \operatorname{map} 1(x, k) \& \operatorname{NOTmap} 2(y, j)$

The latter mapping rule explicitly or implicitly blocks the use of the the first patterning rule with the help of some mechanism still to be formulated. This mechanism is indicated by NOT. Since we use map1 and map2 as meta-predicates stating that there are mapping derivations in corresponding theories encoding linking rules NOT cannot simply be the negation operator. An implementation will be given in section 3.4.2.

The two rule blocks - patterning rules and mapping rules - are then used in communication. Adopting the perspective of the speaker we obtain the following logical sequence of semantic processing ${ }^{2}$ : given a conceptual content (call it communicative sense, cs) patterned as condition (e1,e2), apply the rule for the co-identification of two events to re-pattern the situation, if the rule preconditions are fulfilled, do nothing otherwise. Then map the resulting structure to the syntax using the linking rules corresponding to the patterning used. A hearer must be able to invert the restructuring, of course. S/he would proceed in the following way: s/he would use the standard rule that two expressed events require two expressed arguments.

\footnotetext{
${ }^{2} \mathrm{~A}$ much simpler picture, the more specific rule is always tried first, does not accord with the property of the general rule that it covers the more widespread case. Rule ordering by specificity is by itself indifferent to these consideration, producing the paradoxical situation that if there are very rare special cases, all of them should be tried first nevertheless.
} 
But since there is only one argument, s/he would assume that a more specific rule was used to map the description of the situation to syntax, because $\mathrm{s} /$ he knows that such a rule exists, and as a prerequisite of this rule co-identification of events was assumed. On this account the use of the more specific rule is obligatory, and it must be assumed that more specific rules override more general rules. A variation of this approach is to assume that a more specific patterning rule is directly associated with the verbs which require it, so hearing the verb simply invokes the rule. The co-identification is required by some pragmatic factors, but pragmatics is not directly involved in the rule interaction. Interaction of this kind is a property of default rule systems in general. What is linguistically specific about this inferencing is that this kind of enlightened guesswork is required on the part of the hearer.

Consider deblocking of the more general rule now. From the perspective of the speaker, if the communicative sense has some additional part which serves to distinguish the two events, i. e. to prevent their co-identification, the more specific rule cannot be applied. Note that this piece of inference is also a default rule system: two events which are co-incident in time and space are nevertheless considered not co-identifiable, because of some additional condition, i. e. the rule of co-identification is explicitly blocked to allow the more general rule to apply: the two events remain distinguished and the standard linking rule is used.

If the hearer is to recover the additional condition some reasoning is necessary. S/he must first come to the conclusion that a more specific rule should have been used in the first place. Under the approach where the more specific rule is associated with the verb this is easy. Under the more general approach, where rules are listed independently of the verbs, the situation coded by the communicative sense should be recovered first, and then the mapping possibilities should be tested o establish whether there are more specific rules which should have been applied, but had not been.

The hearer can then recover the additional part proceeding on the assumption that overriding the known requirements must have a reason, if the speaker is sincere, i. e. there must be some additional condition preventing the identification of the two events. Moreover, this recovery has to be not a frequent process, generally applicable to the situation, since if it 
were the the situation would have had alternative analyses, signalling that the rules are unreliable, and a need for another rule would have arisen. In other words, the recovered part of the communicative sense should be not known as a regular part of the situation description, but as an addable, or, as we will say later, abducible part. Abducible parts of situation descriptions may be assumed only if there is evidence for them. Such an evidence is the use of a more general rule in place of a more specific one which is applicable unless an additional condition is assumed. The interaction pattern may be the following: an abducible is added to the precondition of the co-identification rule to form a more specific patterning rule which has no corresponding linking rules but simply blocks co-identification. Then the general pattern is used together with the corresponding linking rules.

Note that this simple reconstruction of Gricean contexts necessitates at least three levels of representation and two mappings between them. Using common terminology the levels might be called communicative sense (the piece where we decide that two events are distinguishable), semantic representation (the pattern condition $(e 1, e 2)$ with additional conditions under which the events can be co-identified) and syntactic structure.

The reconstruction of the the proposal of Levin and Rappaport Hovav in terms of Gricean contexts has some properties which can be exploited to formulate the RSP blocking hypothesis for Russian.

\subsection{The structure of the RSP blocking hypothesis for Russian}

It is then necessary to list some assumptions of the theory in this paper at this juncture, because of their role in making the RSP hypothesis more precise, though they will be discussed in grater detail later.

The correspondence between a communicative sense and a syntactic structure is effected by a set of inference rules based on principles valid in logic. But these rules are only plausible and not inviolable. Computing the inference in either direction characterises both the comprehension and the production of utterances. The computation uses a restricted amount of resources available in the context. We abbreviate this by stating that syntactic structures are interpreted by systems of default rules. An interpretation of a syntactic structure is than a chain of inference steps.

When languages are learned, patterns of such inference steps are learned, 
too. They map communicative sense to syntactic structure and vice versa. The patterns map some semantic relation to some syntactic relation, informally speaking. More precisely, syntactic relations are not interpreted directly, since they are not stated in the language in which inference is defined. Every interpretable syntactic relation gets two corresponding relations in the language of semantic structures. One of them registers whether its corresponding syntactic relation holds in the structure. This kind of relation may also be called syntactic relation, by metonymy, since its corresponding syntactic relation never surfaces in the inference chains. The other correspondent is a technical device. It represents the contextindependent semantic interpretation of the syntactic relation. Such representatives will be called argument relations, because they often hold of the arguments of verbs.

Another assumption, compatible with the recent work in Optimality Theory, in is that initially the learner is unbiased as to which mapping rule is better suited for the grammar, than its possible competitors. The learner may entertain alternatives, unless they do not conflict. If they do, s/he must define her/his preferences.

A language may use some particular means to express the fact that an eventuality is bounded by some state. This can be rendered postulating a conceptual operation of event bounding and its inferential mapping to expressions representing syntactic structures. The mapping of this bounding relation to some syntactic relation is learned in the same way as the other basic rules, i. e. by extracting the linguistic input keys as to which alternative mapping is preferable in accounting for the learning data, given a number of possible alternatives. The sets of alternatives are structured. We assume the following structuring principles in a tentative formulation, which will be revised in section 4.1:

- Uniqueness of realization. A semantic relation may not be realized by two different argument relations at the same time.

- Uniqueness of interpretation. An argument relation should not be mapped to two different semantic relations in the same context.

The two assumptions on structuring principles may be perhaps viewed as characteristic of Gricean contexts, where both the speaker perspective and 
the hearer perspective are important. Since the theory defining the mappings is inferential, the implementation of the two structuring assumptions is based on principles of inference, too.

The structure of the RSP blocking hypothesis is now like this: at the start of learning Russian, both the English kind of inference pattern and some Russian patterns which do a similar job are available. Learning Russian, an individual has to decide on preferences. This happens, because if an English RC-kind of inference is added to the Russian language, it would define an alternative to some inferences realising the boundedness relation in Russian. Russian however has the preference of associating the operation of event bounding with a grammatical relation which is there specifically for this association. In other words, the event bounding operation is grammaticalized in Russian. There arises a conflict between different inference patterns. And since Russian has a preference for the grammaticalized inference pattern, the structuring principles lead then to the exclusion of the English pattern.

To formulate such an approach we need a theory of the semantics of resultatives in English. It should show where the resultative relation between the two events comes from and what kind of relation it is. Then we need a formal statement of the mechanisms of inference involved in Gricean contexts so that we are able to formulate both the inferences in English and in Russian. We also need the inferential implementation of structuring principles. Then we must show what kind of conflict arises, if English inferences are added to Russian.

A word of caution is in order here: the plans articulated above should by no means convey the impression that all the theories mentioned will be invented from scratch. Some proposals will be new. A lot will be borrowed. But because of space limitations the details of the theories which are largely irrelevant for the purposes of this investigation and the discussion of the alternative theories which are left out will be simply omitted in order not to overburden the exposition with general theoretical aspects.

The following section starts the preparatory work of providing the components of explanation. It analyses some relevant theoretical assumptions about the semantics of RSP and discusses in detail a formal semantics of English resultative construction due mainly to Rothstein and Parsons. 


\section{The semantics of RC in English: assumptions and problems}

2.1 Two questions about the notion of result in $\mathrm{RC}$ and the orientation of the analysis

We could propose that $\mathrm{RC}$ arises simply by putting three things together: the relation denoting causation, the event denoted by the verb (causing event) and the event or state denoted by the adjective predicate (caused state). There are different objections to this proposal. The main one is that this simple proposal does not explain obvious constraints on RSP found by Green (1972) in her discussion of instrumental verbs where she discussed among other things acceptability contrasts in pairs like (20).

(20) a. He wiped it clean/dry/smooth

b. *He wiped it damp/dirty/stained

The first question is how these restrictions could be explained. It seems that wiping something implies removing something from a surface, not adding something to a surface. The constraints suggest that the relevant notion of result in $\mathrm{RC}$ has to do with some lexical properties of the verb. We need something like a concept of an expected result of an event of a particular type and expected results could be specified within knowledge which codes this type of event.

The second question is how should a formal semantics reflect this explanation. Let us call descriptions of types of events or event complexes situation schemata. An expected result of an event of a certain type is thus listed in a situation scheme for this type of event. Expected results of wiping something are specified in the situation scheme based on wiping events. A similar proposal was actually made by Georgia M. Green herself. She considers the possibility to have representations like (21) in the lexicon.

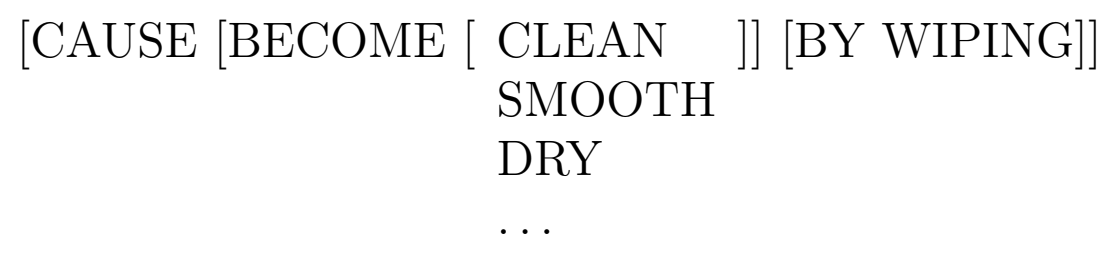

and notes a discouraging property of this proposal: 
The proposal that the semantic representation of instrumental verbs contains a reference to the result predicts for many of these verbs that, given current assumptions about ambiguity, they will be ambiguous between the possible incorporated results. Yet they do not seem to be ambiguous. These verbs seem to exhibit instead a limited sort of vagueness. Yet the question of representing restricted vagueness in lexical entries has never even been raised, as far as I know, much less been answered. And the kind of analysis I have indicated is still essentially arbitrary.

The area of current research interest which goes under the name of underspecification suggests one way of implementing Green's restricted vagueness: separate such things as knowledge about different expected results of an event and knowledge about the general structure of events and of the place these results occupy in the structure. The general structure of the event with a result may be the same for all verbs, but may be further specified by some particular result specification of a particular verb. This approach will be one the major themes of the paper, and the decision to use this underspecification approach motivates in part the choice of Rothstein's analysis for the discussion of the semantics of RC below: it is a formal semantic analysis of RSP which could be used as the most general part of the semantic representation which is always specified with the information from particular verbs. The analysis being formal, some words are due about our formal ontology.

\section{$2.2 \quad$ Formal ontology}

The status of the formal ontology in this paper is rather like that of a model of the hypothetical human model of the world. This formal model is a settheoretic structure, and therefore a disclaimer is in order here. Usually model-theoretic semantics also has a structure it calls a model, but modeltheoretic semantics relates its models directly to to a language. In this paper the model is a representation of cognitive structures in terms of which our language is interpreted and in terms of which its truth conditions are given. Events, states and individuals in this model have properties some of which may seem rather normative, i. e. imposed. Yet these normativeness 
is the price of our cognitive modelling of the world. We assume for instance that all individuals in the model, like events, states and plain objects, build up a universe structured by the part-of relation. This move reflects the hypothesis that such structures are very useful in cataloguing reality. Thus, we postulate a sorted domain of discourse which contains plain atomic and plurality individuals, events and event complexes, states and state complexes ${ }^{3}$ Every one of these sorts is a complete atomic free upper semilattice with a bottom element $\perp$. Thus, every sort is a set $S$ with a partial ordering relation $\leq$ on it such that for all $X \subseteq S$ the least upper bound, l.u.b, $\vee X$ exists ( $S$ is complete), for all $a, b \in S$, if $\neg a \leq b$, then there exists an atom $c$ such that $c \leq a \& \neg c \leq b$ ( $S$ is atomic), for all $a \in S, X \subseteq S$, if $a$ is an atom, and $a \leq X$, then there exists a $b \in S$ such that $a \leq b$ ( $S$ is free). The binary sum operation $\oplus$ which can be defined on these structures is simply the l.u.b of the two operands.

In general we will tacitly assume the set-theoretic apparatus introduced in Krifka (1998). Properties of the events, states, times and individuals in the model will be introduced as the need arises. One important property should be defined at once: an event sum $E$ will be said to be homogeneous under the predicate $P, \operatorname{hom}_{P}(E)$ iff $P(E)$ holds and $E$ can be recursively partitioned into subevents of which $P$ holds, too, all the way to its atomic elements. These assumptions interpreted as a theory of event representation in our cognitive system entail that we can always build a sum structure of any number of eventualities by what we will call generalised conjunction in the next section. More importantly, to accommodate the insights of Levin and Rappaport Hovav we must assume that whether or not an event is analysed as a sum of two events depends at least in part on our intentions. Co-identification blocks such an analysis, for instance, even if it is possible in principle.

We will use a first-order pseudo-code until section 3.1. I this code formulas with open variables will be used as schemata. Their substitution instances will contain discourse referents after the Discourse Representation Theory, e. g., in Kamp and Reyle (1993).

\footnotetext{
${ }^{3}$ We shall adopt the common practice of calling events and states eventualities and will use one sort of variable for the two, $e$ or $E$, where the difference is not crucial.
} 


\subsection{Rothstein's analysis of resultatives in English}

Rothstein (2003) is perhaps the most clear formal analysis of the semantics of resultatives in English. Therefore, though we shall mention others later, we shall discuss hers in detail. It is based on the proposal of how to treat secondary predication in English in general. The resultatives are then only a special case of secondary predication.

Rothstein considers the relation between the primary and the secondary predicates to be based on the generalised conjunction proposed by Laserson (Laserson, 1992). Given two predicates, $\lambda x \lambda e P(x, e)$ and $\lambda y \lambda s Q(y, s)$ generalised conjunction yields

$$
\lambda x \lambda e \exists e 1, e 2(e=e 1 \oplus e 2 \& P(x, e 1) \& Q(x, e 2))
$$

Yet the operation of secondary predication is not pure generalised conjunction, since it reflects the asymmetric role of the predicates. First, the relation requires that the temporal course of the event $e 1, \tau(e 1)$, must be a part of the temporal course of $e 2$. Second, $e 1$ and $e 2$ must share one participant. Given this, the depictive secondary predication illustrated in (22)

(22) John drove the car drunk

would have the interpretation in $(23)$, where $\exp (e)=x$ is something like $x$ is the bearer of state $e$.

(23) $\exists e, e 1, e 2(e=e 1 \oplus e 2 \&$ drove $(e 1) \&$ ag $(e 1)=j o h n \&$ th $(e 1)=$ thecar $\& \operatorname{drunk}(e 2) \& \exp (e 2)=j o h n \& \tau(e 1) \leq \tau(e 2))$

This is the basic proposal covering depictive SP. Resultatives differ from depictives insofar as the secondary predicate, though conjoined with the matrix event, refers rather to its culmination, i. e. the relation of resultative secondary predication is

$$
\lambda x \lambda e \exists e 1, e 2(e=e 1 \oplus e 2 \& P(x, e 1) \& Q(x, e 2 \& \operatorname{cul}(e 1) \leq e 2
$$

To make sense of this theory we should know the properties of culminations. Formally, cul is a function in Rothstein's analysis. For cul to be a function culmination must be unique for any given event, i. e. a culminating event $e$ is then a kind of data structure from which a unique 
eventuality $\operatorname{cul}(e)$ is extracted. If defined, $\operatorname{cul}(e)$ is a near-instantaneous part of $e$ which is its telic point of the predicate making the predicate telic. A telic predicate has the property that if it is true of an event, then all its subevents of which the predicate is also true must be both its initial and its final subevents simultaneously. Removing the telic point destroys this property. ${ }^{4}$.

The discussion in the next section will show that it is better not to make the uniqueness assumption about culminations of a single event. If the notion of function is useful here at all, it is the one of Skolem function, i. e. a device which records semantic dependencies. Nevertheless, we will continue the presentation of Rothstein's semantics employing her tacit uniqueness assumption.

Whenever cul(e1) is defined, according to Rothstein the possible interpretations consist of two cases: the case with the culmination of the matrix event as the left conjunct which produces a resultative reading and the case with the whole matrix event as the left conjunct which produces a depictive reading.

Rothstein assumes that the structure of events which gives rise to resultative interpretation of secondary predication is given by

$$
\exists e, f 1, f 1(e=f 1 \oplus f 2 \& \operatorname{cul}(e)=f 2 \ldots)
$$

where ... specify the relation between $f 1$ and $f 2$. The requirement for the generalised conjunction seems to be that it picks out some event variable which is available in the semantics of VP. A technical solution for this choice is in order here, because verbs which support resultatives are not usually taken to be predicates of three event variables. Suppose we have found a solution. Rothstein's analysis says that here are at least three events which may in principle be available for the summing operation of the generalised conjunction: the event $e 1$ itself, its pre-culmination activity part and its culmination, $\operatorname{cul}(e 1)$. Rothstein suggests that we only have a difference when the whole event $e 1$ is part of the state of the secondary

\footnotetext{
${ }^{4}$ To take an example, if Mary builds a shelf completely, then no proper subpart of the building event which includes the initial event, but excludes its final subevent is also an event of building a shelf completely. Now, removing the event of completion (i. e. cul(e)) destroys this property. The event is merely an event of building a shelf, which now may have differing initial and final subevents (Krifka, 1998)
} 
predicate or its culmination $\operatorname{cul}(e 1)$ is. The reason is that, thought we have two events available in the structure of the primary predication event (i. e. the part which is simply an activity event and the culmination of the main predication event Rothstein calls the becoming event), the activity event can only give rise to a depictive reading which is equivalent to the depictive reading based on the whole primary predication event itself. The event structure under this analysis gets complicated. And we need an answer to the central question of how we get the result component of the RSP meaning. The fact that $\operatorname{cul}(e)=f 2$ holds in $\exists e, f 1, f 1(e=$ $f 1 \oplus f 2 \& \operatorname{cul}(e)=f 2 \ldots \& \tau(f 2) \leq \tau(s))$, where $s$ is a state, does not necessarily express that $s$ is a result of $e$, at least not unless we know more about culminations.

Rothstein sketches the following line of argument in Rothstein (2003):

The structure of an accomplishment involves measuring the progress of an activity in terms of an extended change-of-state event, which has a culmination at which the change-of-state is reached. Constraints on how the activity and change-of-states events are matched make it frequently the case that the change-of-state is cased by the activity, but this is not necessarily the case. The result aspect of the resultative derives from the causal relation and, when it is absent, the result meaning of the resultatives is absent, too.

Verbs with the resultative construction comprise two events, an activity event and a change-of-state event (becoming event), which is associated with the activity event, and which culminates. Its culmination defines the culmination of the whole event referred to by the verb. Note that Rothstein writes

... the resultative does not give the culminating event, which is defined in terms of the incremental Theme, but gives a property of the culminating event.

This property is that of being temporally included in an eventuality which is characterised by the secondary predicate.

Clearly we must infer that the resultative state $e_{r s p}$ is not just any state which happens to temporally contain the temporal trace of the culmination 
of $e$, but a special state, even if the activity event stands in the causing relation with the becoming event. But what makes it special?

Consider the assumptions made by Rothstein so far. The culminations of the becoming events which measure activities are states of the individuals which undergo the change of state. Moreover, the change is conceived as somehow ended at this point. Let us call these c-states. C-states often come about as consequences of the activity events which are measured out. The culminations of the becoming events are instantaneous beginnings of such consequent states. However, it is not explicitly asserted that the state expressed by the secondary predicate has anything to do with the consequent state, only that it includes its beginning. Mere inclusion however need not transfer the causation, hence it need not express any dependency of this state on the activity event.

The resultative interpretation must be due to some tacit reasoning like the following: the inclusion of the culmination with a resultative character into a state $e_{r s p}$ which is specified as dependent on it is always interpreted as asserting the latter state to actually be the c-state (and a consequent state, as the case may be).

This interpretation assumption, if indeed it would be made by Rothstein, requires more discussion and does not account by itself for the restrictions observed by Green. Rothstein does not discuss this aspect of c-states at any length either, so it is impossible to say whether they can be used to provide such an account. The proposal must actually appeal to even more additional reasoning which takes place in the wings, as it were, if cases like (24) are to be accounted for. In such cases the two events of RC do not share a participant.

(24) John sang the baby asleep

Rothstein (2001) gives the following argument for this case. C-states in such cases are rather culminations of implicit becoming events which are not given in the lexicon and must be inferred. The inference is subject to some restrictions. The end part of the implicit event must be homomorphic to the activity event of the primary predication. Only if there is such a correspondence between the event which is responsible for the culmination, i. e. the event of John singing, and the event which culminates in the 
resultative state, i. e. being asleep, are we allowed to use the resultative construction.

We thus have to supply some missing pieces of Rothstein' account. We should at least be able to say what c-states are and why the inclusion of the beginnings of c-states into the state of the secondary predicate induces the latter to be interpreted as a resulting state of the culminated activity. And we should indicate how Green's constraints can be integrated.

There seems to be no straightforward way to fill these two lacunae in Rothstein's analysis. But since the semantics of resultatives proposed by Rothstein draws on the theory of event semantics of Parsons (1990) it might be helpful to have a look at the latter, too. It will be seen that relevant features of this theory raise additional questions about the nature of the final states of the becoming events, since the theory actually has two more kinds of resultative states, one of them is patently a consequent state though not a c-state, and another is very similar to c-states, but is arbitrary and not specified in the verb semantics.

\subsection{Parsons' lexical semantics}

Parsons' neo-davidsonian semantics is based on the notions of events and states. Parsons insists though that sentences denote things different from events or states. Thus, if they denote entities like situations or circumstances, these entities are neither events nor states. There are two distinguished predicates. One of them, Hold, is necessary in any assertion made to the effect that a state holds, the other, Cul is necessary in any assertion that an event occurs and culminates in something. Cul $(e, t)$ states that an event culminates at time $t$. Parsons provides only some intuitive hints as to how the notion of culmination is understood. He notes that an event often has a development portion and a culmination, but in fact, Parsons' semantics has no notion of culmination which exactly matches that of Rothstein. The relevant context distinguishing them are progressive forms: eventualities interpreting progressive forms have a culmination in the sense of Rothstein, because they allow secondary predication, but they do not culminate in the sense of Parsons. The treatment of the English progressive by Parsons assumes that the eventuality described by the verb in a progressive form is treated as a state. The notions involved in the 
analysis of RSP should therefore differ somewhat under the two accounts, since we are no longer allowed to say that RSP amounts to the culmination of the becoming event being included into the SP-state.

Apart from this difference Parsons distinguishes two, obviously also resultative, relationships between culminating events and states. He assumes that

For every event $e$ that culminates, there is a corresponding state that holds forever after it. This is "the state of e's having culminated which I call the "Resultant state of e", or "e's R-state"...

It is important not to identify the Resultant state of an event with its "target" state. If I throw a ball onto the roof, the target state of this event is the ball's being on the roof, a state that may or may not last for a long time. What I am calling the Resultant-state is different; it is the state of my having thrown the ball onto the roof, and it is a state that cannot cease holding at some later time.

Note that R-states hold regardless of whether target states hold. If I say I have thrown the ball onto the roof as an answer to the question of the headmaster Who has thrown the ball at me?, the ball never reached the roof. The target state does not hold, though specified, but the Resultant state was initiated and therefore holds.

Parsons claims that the relation between culminated events and their $\mathrm{R}$-states is given by the principle(25).

(25) e's R-state holds at $\mathrm{t} \equiv$ e culminates at some time at or before $\mathrm{t}$

This principle allows Parsons to show the equivalence of simple past and present perfect in some contexts, cf. (26).

Mary has eaten the apple

For some event e:

(26)

e is an eating

the agent of e is Mary

the theme of e is the apple, and

e's R-state holds now
Mary ate the apple

For some event e:

e is an eating

the agent of e is Mary

the theme of e is the apple, and

e culminates before now

R-states are unique and it is possible to introduce the notation $r s t(e)$ to denote the function which picks out the R-state of the event $e$ and a 
predicate rstate $(s, e)$ asserting that $s$ is a R-state of $e$. Note that R-states are not necessarily c-states.

Target states, which will be denoted by t-states will be discussed somewhat later.

Parsons provides a brief discussion of RSP, which he terms resultative tags. R-states and target states are not used in the analyses of RC, notwithstanding their resultativity, and his proposal amounts to a conventional two-event analysis of RSP. One of the events is the event denoted by the verb, the other is the event of becoming, i. e. of gradually acquiring the state denoted by the SP-adjective. The causative relation is explicitly asserted to hold between these two events. The analysis has therefore the known drawback of being indifferent to lexical information. With this, we are back with the analysis by Rothstein as a better option.

Now we have three different kinds of state which seem to involve some notion of result. Two of which seem to play a role in RC: c-states and target states (t-states). R-states which we will write as r-states, are not associated with RC. Naturally, we might ask whether culminations of becoming events in the sense of Rothstein may initiate r-states. RSP-states surely play a role in defining r-states, i. e. if we choose a present perfect form of RSP, we assert that there is an r-state such that the c-state and the RSP-state hold in he situation which covers the time of utterance and this r-state holds forever. The PP-RSP is well attested, however. Yet though we now have two kinds of consequent states we are not much better off with c-states, nor with t-states.

\subsection{A normative view of events: Moens' eventuality scheme}

Perhaps relating a culmination and a state via the notion of result reflects some characteristics of our conceptual language-supporting ontology. Very similar proposals have already been made in similar contexts. Thus, as noted by Krifka (Krifka, 1998), discussing the notion of telicity:

... the distinction between telicity and atelicity should not be one in the nature of the object described, but in the description applied to the object. 
... For example, one and the same event of running can be described by running (i. e. by an atelic predicate) or by running a mile (i. e. a telic, or delimited, predicate).

Adopting this perspective, what kind of event description is employed in RSP? Consider the idea put forward in Moens (1987), which may be relevant to this question:

It seems, in fact, that the meaning of all the categories in the aspectual network is associated with a complex entity consisting of a preparatory process, which can lead up to (without necessarily reaching) a culmination point, and this in turn has certain consequences attached to it. It can be represented pictorially as follows

\begin{tabular}{l|l} 
preparatory process & consequent state \\
\hline &
\end{tabular}

culmination

We now consider this scheme, which will be called eventuality scheme, to be a theoretical construct of human ontology which structures the semantic contribution of the verb to the semantics of a sentence it is in. Viewing possible consequences we distinguish one special set of them. We consider the scheme as stating, in particular, that any event we choose to describe can be described as bounded by a state, with the bounding state being in the set of chosen consequences. In this sense each event may have a culmination. This is an assumption about the existence of patterning rules, similar to the one made by Rappaport Hovav and Levin.

Applying the eventuality scheme to the analysis proposed by Rothstein we get the following description: the culmination of the event $e$ consisting of the activity event and the becoming event initiates a bounding consequent state. This state bounds the culminated event $e$. Eventuality scheme does not define particular culminations, it only makes a provision for one, stipulating that events may be described as bounded. But it must specify the general properties of culminating events. Any event which is described as culminating must have these properties.

Some of the the characteristics of this notion of (bounding) culmination are similar to those required by Parsons and Rothstein: the eventuality scheme treats culmination as a point specifying that it falls within the 
beginning of the consequent state. It is also at least included in the end point of the culminating event, hence asserts the existence of the endpoint. But it depends on the nature of the bounding event.

Any event may have different culminations specified with the help of the bounding state. We follow Parsons, for instance, in assuming that any event that is described as temporally bounded has a culmination point, i. e. the one defined by the temporal bound. The event is simply described as culminated after having reached this temporal bounding point. Of course, it is also possible that some intrinsically given part of the event may serve as an expected culmination. The part of a painting event which contains finishing the painting of a surface is an example. In other cases the culmination can be specified externally, like in case of running. The culmination may be either defined as running a specified distance or for a specified time and in case of throwing the ball onto the roof this may be the point where the ball arrives on the roof.

Now, a look on the three states which were distilled after having discussed the approaches by Rothstein and Parsons suggests that c-states could be characterised as being the bounding consequent states of expected culminations whereas t-states are bounding (possibly consequent) states defining explicitly given culminations, where the culminations are initial points of c-states and of t-states and final points of the becoming events or of processes. Expected culminations are lexical knowledge, so their definition varies with the verb: the expected culmination of reading a book is having read the book to the end, that of cooking a fish is having cooked the fish to some well-defined state. T-state culminations are imposed rather then expected or, if expected, at least provide a parameter which is fixed sentence-internally but not lexically.

These two kind of states differ naturally from r-states, and we shall comment on the difference in a moment.

It should be emphasised that there is a major difference between an ontologically defined notion of a culmination of Rothstein's approach and a prescriptive one which we now adopt. Just like in the example concerning telicity by Krifka above, we may classify an event of running as having no culmination under Moens' scheme or as having a culmination, because it was an event of running two miles. So whether or not an eventuality 
has a culmination depends on its description. Also, an event may have different culminations, if different descriptions are possible. Rothstein's culminations are a subclass of ours. But we must give up the uniqueness assumptions for culminations. Thus, if we may use $\operatorname{cul}(e)$ at all, then only as a kind of Skolem function, denoting some concrete function kept fixed in the context. In sum, we arrive at the following patterning predicates for one particular case of the eventuality scheme

$$
\{\operatorname{proc}(e), \operatorname{bound}(e, s t)\}
$$

where bounds $(e, s t)$ underlies a particular case of the eventuality scheme. The predicate which could render Moens' more general notion of consequences will be called consstate. We shall have more to say about the bounding operation in section 4.2. But we should note here that neither expected bounding states (c-states) nor t-states necessarily assert that a result state holds in the situation. He is drawing a circle does not assert that the circle has been drawn. Similarly, He is painting the wall red does not assert that the wall has been painted red, nor does He is running into the wood mean he has reached the wood. It seems that even if the resultative component of meaning is present, the results themselves are outside the situation.

The utility of the normative culmination construct is to provide inferences about the event over and above the assertion that it occurred at some time. But if such inferences are to be consistent they should be cancellable in some way, since it is hardly useful to be able to infer about an event that it both culminates and does not culminate. A plausible way of implementing this is to use contexts. If one and the same event may or may not be viewed as having a particular culmination depending on a description, choosing a description establishes a context which determines a set of properties which cannot be revised, as long as the context is valid. All inferences then must take place in this context. Note that on this approach the extension of the predicate $C u l$ of Parsons must vary with contexts. Since it is obvious that explicit descriptions of culminations may be provided by different parts of sentence, the relevant notion of context will surely have something to do with the notion of situation, since sentences denote situations. Moreover, situations may or may not include realized expectations. The notion of situation is therefore important and 
should be discussed in more detail.

\subsection{Situations}

\subsubsection{General properties of situations}

We continue assuming with Parsons that situations are denoted by sentences. Yet we are actually more interested in their role as information providers for inference during communication. A situation as the term is understood here is a device to structure and communicate information about the world. The basic assumption about the inferential role of situations deserves a special name.

\section{(27) Anchoring to situation}

All reasoning depending on an assertion is done in the context of the situation about which the assertion is made

We will thus use the notion context of the situation, ct $(s)$. This context largely determines the inferential potential which is provided by the situation. The postulate should be taken to imply that the inference steps are defined within this context, but not across its bounds, unless there are rules of transcending them, and that any assertion is made relative to the reference time of the situation and to its temporal bounds in general. The context of a situation thus specifies its inferential resources. A suitable theory of context was proposed by McCarthy and Buvač (1998). It will be discussed in more detail in section 3.5.2.

A description of a situation includes, among other things, a situation referent, say $s$, a reference time of the situation, $r t_{s}$, a time course of the situation $\tau(s)$ with the general condition $r t_{s} \in \tau_{s}$. The reference time of the situation specifies which portion of the the eventuality scheme is being considered relevant. In this way we integrate the assumption that even intrinsically culminating events like drawing a circle may be considered as not culminating within the bounds of situation.

Situations in their role as contexts of inference should provide general templates for eventuality schemes. Two seem rather plausible, cf. (28), (29):

(28) $\operatorname{situation}(s, e 1, r t(s), \tau(s)), \operatorname{proc}(e 1, s)$ 
The type (28) might be a basis for the English non-perfective situations.

$$
\begin{aligned}
& \operatorname{situation}(s, e 1, e 2, t, r t(s), \tau(s)), \operatorname{proc}(e 1, s), \\
& \operatorname{overlaps}(e 1, e 2, t), \operatorname{init}(e 1, e 2), \text { consstate }(e 1, e 2)
\end{aligned}
$$

If event $e$ terminates at time $t$ and state $s t$ holds at or immediately after $t$, but not before it, we will say that $e$ initiates st, init $(e, s t)$. We will call $t$ the initial point of st wrt. e. The condition overlaps $(e 1, e 2, t)$ fixes the initial point of the consequent state and hence the time of the culmination. We do not want to specify any particular kind of resultative relation between the event $e 1$ and its consequent state $e 2$ at this stage, but merely that the latter is always initiated by the former. If an eventuality culminates in situation $s$, the situation template should be extended with $\operatorname{cul}(e 1, t, s)$, but in general we would not like to always have this predicate in the situation template. Similarly for hold.

\subsubsection{Situation frames}

The discussion proceeded up to now on the assumption that specifying a situation draws on two different resource kinds: on our general conceptual knowledge and on patterning rules.

Consider our general knowledge as it is being packed into a situation. We know, for a lot of processes, what their expected course is and what the results of this course are at different phases of the process, e. g. we know that continuing reading brings us to the end of the text, if the latter is finite, and continuing frying a pork chop after some point at which it is still edible burns it to ashes. The mapping of the knowledge of this level of generality to syntactic structure does not proceed directly. If this knowledge constitutes communicative sense, we communicate it making use of an intermediate structure of a higher order of generality, i. e. a situation template. I will use the terms situation scheme and situation frame to distinguish between communicative sense and more general structuring devices. In doing this we simply continue to explore the assumed property of Gricean contexts which separates conceptual knowledge from structuring rules and the latter from linking rules. Situation schemata constitute communicative sense. The distinction allows us to express the communicative sense that though there is an expectation that given some natural 
course of an event the event is bound to end and initiate a certain state, it does not actually end and the state may remain a potentiality. The part of the statement dealing with expectations would involve a situation scheme, the part about actual circumstances involves a situation frame.

The idea of two levels of specification dictates the following general algorithm of computing a syntactic structure from communicative sense and vice versa. Generating texts proceeds via choosing a suitable situation frame for the situation scheme which patterns the communicative sense and then mapping this situation frame to syntactic structures. And conversely, interpreting a syntactic structure amounts to finding a situation frame suitable for the syntactic structure and specifying it in accordance with pragmatic and semantic principles by finding a necessary situation scheme. In this algorithms the eventuality scheme is associated with the situation frame, which may also reflect additional properties of the sentential syntactic structure.

It is customary to distinguish two parts of the situation for the purpose of mapping it to syntax. The dichotomy reflects a number of distinguishing properties of the participants of the situation, which are reflected in classifications like direct arguments vs indirect ones. The dichotomy shows up also in some treatments of case, e. g. Jakobson's distinction between the core and the peripheral cases. We will take up this distinction as a heuristic means, without justifying it here, and assume that one part of the situation scheme is structured by a situation frame. The part of the situation scheme which is structured by a situation frame will be called situation core. The remaining information in the situation scheme will be called situation frame adjuncts or sfadjuncts for short.

Consider the following partial sframes for the sake of illustrating the assumptions:

(30) 1. A partial perfective frame

$$
\begin{aligned}
& \operatorname{proc}(e 1, s) \\
& \operatorname{cul}(e 1, t, s) \\
& \text { holds }(e 2, s) \\
& \text { consstate }(e 1, e 2) \\
& \text { rstate }(e 2, s) \\
& \operatorname{tr}(s) \subset e 2
\end{aligned}
$$


The situation is viewed from the standpoint of the r-state of the preparatory process event.

2. A partial progressive frame

$$
\begin{aligned}
& t_{r}(s) \subset e 1 \\
& \operatorname{proc}(e 1, s) \& h o m(e 1)
\end{aligned}
$$

The situation is viewed from the standpoint of the preparatory process event itself. Preparatory process is always homogeneous. The requirement is consistent, because the frame does not specify that the process culminates in the situation.

The frames reflect some analyses of the perfect (Jespersen, 1924; Kamp and Reyle, 1993) and the progressive (Krifka, 1992) in English.

The ingredient of situation frames we are most interested in is the consequent state.

\subsubsection{Consequent states and bounding states}

In the two RSP analyses reviewed above we encountered three kinds of state: c-states, r-states and t-states. Both c-states and t-states were then grouped into the set of bounding states. We will now relate them to the notion of consequent state. To do this we need the notion of result which could characterise the consequent state of the culminated preparatory process as a set of consequences.

We assume that eventualities can be associated with a single bearer individual, however complex, regardless of how many other event participants there may be. Such a choice seems to be always possible and determines the viewpoint under which the event is seen. Tenny (1994), discussing examples like translate a poem and the the role of objects in characterising such events by measuring them out, notes that "Not only do these events have temporal bounds, but these bounds are provided by the referent of the internal argument. In other words, it is the object which delimits the event.". This individual provides at least one property $P(x, e)$ which is considered to be constitutive for the eventuality of which it is the bearer. The consequent state makes use of this property of the bearer.

On a pre-theoretical level a result of event $e$ is an eventuality, i. e. an event or a state, $s$ which has at least the following characteristics: 
(31) a. $s$ is pre-dated by an event $e, \operatorname{init}(e, s)$, in the sense that there is a time point before or at which $e$ culminates but $s$ does not hold and after which $s$ holds;

b. the association between the event $e$ and the result state $s$ has some systematicity. We would expect that if we keep all the relevant conditions which are true during $e$ and replicate this event, we expect to get the same kind of state;

c. there is some not just statistic connection between the two. Perhaps, considering $s$ to be a result of $e$ we would want to be able to produce some explanation of why we think it is so. If my carelessness led to a broken vase it is expected that it is possible, e. g. to provide a causal chain between the two eventualities;

A working partial definition of a consequent sate can be then as in (32), for some parameter $Q(e)$.

(32) A consequent state of $e$ relative to $Q(x, e)$ is a state $s$ characterised as $P(x, s)$ with $x$ the bearer of $e$, such that

(i) $[Q(x, e) \& \operatorname{init}(e, s) \& P(x, s)]$ and

(ii) (31b) and (31c) hold, abbreviated as cause $(e, s)$, i. e.

$$
[Q(x, e) \& \operatorname{init}(e, s) \& P(x, s)] \& \operatorname{cause}(e, s) \rightarrow \operatorname{consstate}(e, s)
$$

In this definition the bearer of the event and the state $x$ is the individual by virtue of which the event referent and the state referent are in the situation at all. ${ }^{5}$ The parameter $Q$ determines which kind of events yre considered to produce the state with the property $P(x, s)$. Note that the definition may be used to characterise which states are eligible as consequent states, given $Q(e)$. Thus, frying a steak to a point when it is well done is one kind of consequent state of frying a steak; frying it beyond this point is also a well-defined consequent state, as is frying it less then well-done.

\footnotetext{
${ }^{5}$ Notice that neither init $(e, s)$ nor cause $(e, s)$ are complete representations of the notion of result by themselves. There might be pairs $\langle e, s\rangle$ satisfying the predicate $\operatorname{init}(e, s)$ where we would hesitate that the state is a result of the event. Take, for instance, Newton's apple. We probably would not like to describe Newton's discovery of the law of gravity as the result of the apple fall, given our (32). On the other hand, the notion of result as defined here is tighter than simply a causal chain. This definition is tailor-made to reflect the notion of result as relevant to the portion of semantics analysis under discussion.
} 
Whether or not these consequent states are grammaticalized in a language is a property of a particular language. Russian, for instance, makes such distinctions between crossing vs. not reaching some desirable point via the prefixes pere- and nedo-.

Consider again some properties of three kinds of states which allow them to be viewed as consequent states, given this definition.

1. C-states The c-states from the treatment by Rothstein share a participant with the event, are initiated by it, must stand in some kind or resultative relation to the event. The discussion of the semantics of $\mathrm{RC}$ suggests that c-states are more or less expected results of culminating events $\operatorname{cul}(e, t, s)$. The events are expected to culminate given some natural course of events. With this characteristics it becomes obvious that c-states are defined at the level of representation which reflects our general knowledge about classes of eventualities, reflecting lexical dependencies as desired. An appropriate level of generality would be our situation schemata. Since a bounding c-state, however, may or may not hold in the situation, the resultative reading arises only if the situation frame contains the predicates hold and cul for he corresponding state and event. If the predicate is not there we have only a prospective result.

2. R-states The r-states from the treatment by Parsons also have some important properties of the consequent states. They share at least one bearer, are initiated by a culminated preparatory process and stand in the resultative relation to the event. They hold if at all, then forever. This clearly distinguishes then from c-states and t-states. The perfective frame together with this persistence property requires that if they figure in the description of the situation, they must hold in it. R-states are thus very typical consequent states. Their another distinguishing feature is the dependence on the structure of the assertion as a whole, and not only on the lexical information about the type of preparatory process. One of the major inferences is existential persistence within a situation, clearly a non-lexical notion in general. Consider, for example, a description of a r-state given by Thomson and Martinet (Thomson and Martinet, 1960, p. 106 of the second edition, 1969) 
'I have seen wolves in that forest' implies that it is still possible to see them, and 'John Smith has written a number of short stories' implies that John Smith is still alive and can write more. If however the forest has been cut down and John Smith is dead we would say, 'I saw wolves in that forest once/several times' or 'I used to see wolves in that forest' and 'John Smith wrote a number of short stories'.

Yet another difference is that these states clearly do not provide bounding points for events, cf. I have run.

3. T-states. The target or t-states from the treatment by Parsons initiated by an event are conceivable as results of this event and also share a participant with this event. In contrast to c-states they are not specified lexically in the situation scheme, though they are states of a participant. They are only intended to be viewed as results of these events, defining the culmination of the event. Throwing a ball onto a roof can naturally end in the ball being on it, before it, or somewhere above or behind it. T-states are states that explicitly define where the event of which they are a consequent state should be considered to end, given that they hold. In other words, they need not be associated with a natural course of the event they bound and are specified extrinsically, generating a plethora of different culminations. If they hold in a situation, they also qualify as consequent states. The abovementioned prefixes of Russian pere- and nedo-, which specify a point or an interval on some dimension and indicate that the relevant region is after or, respectively, before this point, provide good examples of lexicalisations of t-states.

Thus, all the three kinds of states discussed are special cases of consequent states, but two of them may happen to be only prospective results. Of the three kinds of consequent states only two are associated with RSP in English. The third one, r-states, are grammaticalized in a different way, i. e. by a perfect verb form. 


\subsection{The bounding operation}

It thus seems to be possible to group c-states and t-states under the name of bounding states and postulate an operation of event bounding. We consider event bounding to be a language-independent conceptual operation which may or may not have a specialised linguistic realization (i. e. be grammaticalized or not). The following is a short sketch of what a theory of the bounding operation should contain ${ }^{6}$.

We store information about the world partly in chunks which may be thought of as very general situation descriptions based on event ontology. Now, at least for activities, such description can contain designated points characterised in terms of the state of some selected event participant which we will term event bearer. Such selected states could reflect different attitudes of the agent, different stages of the activity, its natural termination points, points relevant for planning, etc. Whatever they are, they may provide some information which is worth being systematically mapped to syntax. The event bounding is then an operation which is defined in general terms, but specifiable for more concrete cases, and used in mapping additional information about events in the situation to syntactic structure. The essence of the bounding operation seems to be this: we choose a dimension as some measurable or order-scaled property of the bearer of the event. We conceptualise a state - a point on a scale of this property and provide some criteria distinguishing it from other points on this scale, forming thus the bounding state of the event in the context in which the choice was made. If the state is asserted to hold in the situation, we then require that the event is viewed as terminated at this point and that it initiates the bounding state.

We assume that such an operation exists. An obvious question is where do the dimensions come from? Let us consider c-states. If the distinguishing criteria are statable in terms of an expected, typical result of the activity, these particular bounding states are what we referred to as cstates in Rothstein's analysis. But there are other possibilities. Since the event bounding is freely available, an activity may even get a designated state which is rather vaguely specified. One option, already indicated, is a

\footnotetext{
${ }^{6}$ See also Tenny (1995) for more discussion of measuring out and boundedness.
} 
natural expected terminal point of the activity. Eating an apple terminates naturally when the apple has been eaten. This is the expected termination dimension given for consumed incremental themes. Incremental themes for creation verbs define natural termination points at which the thematic object has been created or completed.

Apart from natural termination points we might note semi-arbitrary internally defined points like acquiring a certain form, covering a certain path, etc.. Both are subcases of what we called expected results. The resultative relation is indeed there, so they automatically qualify as consequent states.

Dimensions might be imposed somewhat more externally to the activity, if they are homomorphically related to it, as noted by Rothstein. Thus, drinking has no natural dimension which measures stupidity. But a designated point of drinking may be associated with some state of the drinker, in He drank himself silly/stupid.

Internally defined designated points obviously define verb classes. Externally defined designated points need not. But they may depend on the characteristics of event ontology. Thus, if an event is protracted on some dimension which may be conceived as isomorphic to (one-dimensional) space, space-oriented selection of designated point is possible, cf. Walked into the garden vs Talked himself into the hearts of the audience.

The interesting feature of event bounding in English is that the dimensions are not systematically associated with any grammatical device. Particles can sometimes mark the presence of a dimension, but their interpretations are usually lexically determined, cf. call up vs. fill up vs. sit up.

Let us return to Rothstein's idea that culminations of an activity are given in terms of a change-of-state event. The notion of dimension formulated and discussed above seems to be compatible with Rothstein's analysis. A culmination of the change-of-state event is then the point of intersection of a designated state with the activity event, i. e. an outcome of applying event bounding to the activity. The bounding dimension is the property which defines the change-of-state event. In other words, the bounding operation may use c-states for bounding states.

As noted, the relation between the RSP event $e_{r s p}$ and the bounding 
c-state is that of inclusion, and therefore does not allow us to speak of the resultative reading of RSP. The resultative reading of a secondary predicate can arise on this account from some kind of identification of bounding event with $e_{r s p}$. But what kind of identification is this and why is it possible? Note that in principle the two events need not be co-extensive. If a door is painted red, it may still remain painted after some time, but be no longer red. Nevertheless, the bounds of the situation seem usually to be set in such a way that the two states are co-extensive within the situation. We thus may attempt to identify them in the context of the situation.

Co-identification is then another conceptual operation. Since we follow Levin and Rappaport Hovav in assuming it, we will not attempt to justify its existence. However, it should be noted that it has additional requirements in our case. The properties of events being co-identified must be compatible. Consider He has pumped the tyre flat. It is generally assumed that He has pumped the tyre entails The tyre is pumped up in the same situation, so a conjunction with two contradicting conjuncts must always be false. However, this sentence sounds like it would violate some expectation, and therefore invites for an additional interpretation restoring the consistency which is expected to cancel only the first conjunct. The second conjunct is assumed to hold true under such restored-consistency interpretation. Simple identification of states does not reflect this expectation.

The notion of compatibility involved in the mechanism of identification can be made a bit more precise. Two cases may be distinguished.

If the resultant state introduced by the secondary predicate implies the c-state bounding point of the primary predicate the two states are coidentified. If the SP-state contradicts the default bounding point, the result of co-identification is unacceptable, e. g. wipe the table spotted, but some additional reasoning in a Gricean context may redefine the dimension, similarly to the second case below.

If the state introduced by the secondary predicate does not imply the default bounding c-state of the primary predicate but does not contradict it, or if the primary predicate has no default bounding c-state a new dimension is chosen based on the state of the secondary predicate. This adoption of a new dimension is hypothetical and subject to plausibility reasoning. If this dimension does not sufficiently characterise the activity expressed 
by the primary predicate, the result is infelicitous, since the hypothesis is implausible. Once it is plausible, the identification takes place in reverse direction, so to say, and the activity acquires sort of a new expected result.

Consider some variations of Rothstein's example which seem to support the proposal. It is often the case with non-goal oriented activities which have no intrinsic points of interest, like barking, that they lack bounding state candidates. In this case we add a new dimension to accommodate the SP. The felicity of the sentences depends on measurability the dimension imposes on the activity, and probably on nothing else. Unless there is no contradiction it seems that everything goes, cf. Every night a dog barked me to sleep/angry/back home.. Similarly, the case of He has pumped the tyre flat is easily explainable: the asymmetry in the treatment of the conjuncts is due to the fact that the second conjunct provides an alternative dimension. Note that we obviously require Gricean contexts in order to perform this reasoning.

There is an objection at this point which we have to address. Rothstein's claim was that in case an external change-of-state event is used to measure the process event we do not necessarily have a resultative reading. Since our treatment considers all instances of RSP be instances of the consequent relation frame and therefore to exhibit at least some weak causality, we contradict her.

Examples which lead Rothstein to her conclusion that the resultative component may be absent in RSP are like (33)

(33) The audience applauded the singer off the stage

The sentence allows indeed a non-resultative reading. However, this reading is not systematic. Thus, it is impossible to have a similar reading in (34)

(34) He accompanied me tired

If there is a felicitous interpretation of (34) at all, it is a resultative one. The conclusion we draw here is that a non-resultative reading is sometimes possible as parasitic on the resultative, if the causative component is very weak, but that a causative component is present nevertheless.

Now, equipped with these notions we may return to the analysis of RSP by Rothstein to fill in the lacunae. The inclusion of the beginnings 
of c-states into the state of the secondary predicate does not induce the latter to be interpreted as a resulting state of the culminated activity. The resulting states are already specified lexically. What we should explain is how the two states, the c-state and the state of the SP, are related. The restrictions on RSP noted by Green are taken care of, since c-states are bounding states.

\subsection{Bounding and co-identification in RSP in English}

In this section we will go through an English example to illustrate how the notions we discussed in the preceding sections interact to produce a modified Rothstein semantics for RSP in English. We will use formulas with open variables as schemata which are to be instantiated by discourse referents, set notation to represent conjunction, and skip all inessential technical details, e. g. variable management. Though the formulas are to serve as defaults, the formalisation of defaults will be postponed till the next section.

The core idea for the treatment of RSP in English is that the resultative reading arises from the co-identification of the eventuality of the secondary predicate with the c-state of the situation scheme. But this co-identification is restricted in its effect to the context of the situation about which the assertion is made. This provision requires some notion of context, which we will provide later. Remember that according to our interpretation of Rothstein in terms of Moens' eventuality scheme we have three eventualities in a situation scheme:

- the process event part

- the becoming event

- the event bounding state of the bearer of the becoming event, which is the final part of the becoming event, overlaps with the end of the process event and is initiated by it.

Consider now a tentative partial situation scheme (36) for the verb paint in(35).

(35) John painted the door 


$$
\begin{aligned}
& \text { paint }(e), e=e_{p r} \oplus e_{b e c}, \text { cause }\left(e_{p r}, e_{b e c}\right) \\
& \text { spreading_paint_over }\left(e_{p r}\right), \text { agent }\left(x, e_{p r}\right) \\
& \text { (36) } \text { getting_covered_with_paint }\left(e_{b e c}\right), \text { theme }\left(y, e_{b e c}\right) \& \operatorname{surface}(y) \\
& \text { expected_result_paint }\left(e, e_{b}\right) \\
& \rightarrow \text { end }\left(e_{b e c}\right)=e_{b} \& \operatorname{init}\left(\operatorname{end}\left(e_{p r}\right), e_{b}\right) \& \text { covered_with_paint }\left(e_{b}, y\right)
\end{aligned}
$$

Here $e_{p r}$ is the process part, $e_{b e c}$ the becoming event and $e_{b}$ the bounding event, which is also an expected result of painting, hence a c-state. The choice of the eventuality referent which leads to the interpretation of SP is free. An implementation of this free choice in terms of abduction will be shown in (66). Only two of them are directly accessible through their sum, though. This means that, although we know what the expected result of painting is, the scheme by itself does not tell us which parts of it hold in the situation $s$. In particular, it will not automatically associate the result state with the event in a situation.

It seems though that there is an implication in (35) to the effect that the event culminated in the situation and the painted surface is covered with paint. This information is not supplied by a situation frame according to the theory which is being developed here. Where could the implicature originate from?

The important part of the situation frame employed in (35) is rather like (37), since Parsons' theory requires that the preparatory process culminated, if the event was entirely in the past.

$$
\begin{aligned}
& \operatorname{situation}(s, e, r t(s), \tau(s)) \& \operatorname{proc}(e, s) \\
& \operatorname{cul}(e, s, \operatorname{rt}(s)) \& \text { before }(\tau(s), \text { now })
\end{aligned}
$$

According to the frame the time course of the situation, and hence its reference time, are in the past. The culmination was achieved within the situation. Clearly then the c-state of the event holds in the situation, too. A reasonable assumption is that the implicature is associated with English simple past tense form, hence this situation frame might be English specific, but need not hold in general. In other words, English allows us to make an assumption to the effect that if an event culminated in a situation, the expected result of the event holds by default in this situation, too. But note that the relation expected_result_paint $\left(e, e_{b}\right)$ is not mapped to the syntactic structure. This is plausible, since the resultative impli- 
cature involves individual verbs. If a situation scheme has no resultative component, such a component is not added by the implicature, e. g. John sang and I listened has no implication that John was singing something to the end.

Considering the operation of bounding, it is plausible to assume that it can in principle be associated with the syntax. Hence, if it connects the event-bounding state (the expected result of painting) and the consequent state of the situation frame, the resultative component of the situation scheme may get a realization in the syntax as a consequent state. The necessaryly connection clause could look like (38).

$$
\begin{aligned}
& \text { expected_result_paint }(e 1, e 2) \rightarrow c_{-} \text {state }(e 1, e 2) \\
& \text { c_state }(e 1, e 2) \rightarrow \text { bounds }(e 1, e 2) \\
& \text { bounds }(e 1, e 2) \rightarrow \operatorname{consstate}(e 1, e 2)
\end{aligned}
$$

At the moment we simply conclude that English does not provide a grammaticalization for the conceptual operation of bounding.

Collecting the pieces we have now a tentative representation of the situation in (39).

$$
\begin{aligned}
& \text { situation }(s, e, r t(s), \tau(s)) \& \\
& \operatorname{proc}(e, s) \& \text { cul }(e, s, \operatorname{tr}(s)) \& \\
& \text { before }(\tau(s), \text { now }) \& \text { holds }\left(e_{r}, s\right) \\
& \text { paint }(e) \& e=e_{p r} \oplus e_{b e c}, \text { cause }\left(e_{p r}, e_{b e c}\right) \\
& \text { spreading_paint_over }\left(e_{p r}\right), \text { agent }\left(j, e_{p r}\right) \\
& \text { getting_covered_with_paint }\left(e_{b e c}\right), \\
& \text { theme }\left(t h e \_d o o r, e_{b e c}\right), \text { surface }(\text { the_door }) \\
& \text { expected_result_paint }\left(e, e_{r}\right) \\
& \rightarrow \\
& \text { end }\left(e_{b e c}\right)=e_{b} \& i n i t\left(e n d\left(e_{p r o c}\right), e_{b e c}\right) \\
& \& \text { covered_with_paint }\left(e_{b}, \& \text { the_door }\right) \\
& \text { expected_result_paint }\left(e, e_{r}\right)
\end{aligned}
$$

The last expression is assumed in order to accommodate the implicature associated with the simple past tense in English. Exactly what the particular implicatures of different verbs are is beyond this paper. The formalisation of the assumption procedure, however, will be presented in the next section. 
Let us turn to an informal discussion of RSP now, e. g. (40), to see what happens with the resultative component of the situation scheme there.

(40) John painted the door red

As noted in the discussion of Rothstein's proposal, a technical solution for the access to the culmination variable was necessary. The situation scheme provided by the verb makes two eventualities available. It may be assumed that the secondary predicate is free to choose any one of them. The contribution of the predicate is to (39) approximately as in (41).

$$
\begin{aligned}
& e_{\oplus}=e_{b} \oplus e_{\text {res }} \\
& e_{b} \leq e_{\text {res }} \\
& \text { red }\left(e_{\text {res }}\right) \& \exp \left(e_{\text {res }}, \text { the_door }\right)
\end{aligned}
$$

Again, we do not bother about the technical details now.

The idea how to patch the proposal of Rothstein is to assume that the two events, $e_{b}$ and $e_{r e s}$, are co-identified. To explain the co-identification assume further that if one conjunct of the generalised conjunction is dependent on the other for some of its relevant properties, and the first is a c-state, the second is assumed to also be a c-state. This in turn might be a sufficient reason to assume that it is included in the first within the bounds of the situation, if they are of the same semantic sort, since we do not know its temporal properties in isolation. Dependent on this inclusion it seems to be possible to co-indetifiy the two in the situation. The reasoning steps for secondary predicates are thus

1. we know $c \_s t a t e\left(e, e_{b}\right)$, and $e_{b} \leq e_{r e s}$

2. we know that the relation $e_{\oplus}=e_{b} \oplus e_{\text {res }}$ interprets SP

3 . we assume then that $c \_s t a t e\left(e, e_{\text {res }}\right)$

4. hence, we may assume that $e_{r e s} \leq e_{b}$

5 . hence, sametime $\left(e_{b}, e_{r e s}\right)$ sameplace $\left(e_{b}, e_{r e s}\right)$ hold in the situation and therefore

6. co_identify $\left(e_{b}, e_{\text {res }}\right)$ holds

Note that co-identification along these lines could plausibly explain three things: the implicature that the SP states tend to imply the expected result states, the restrictions observed by Green as well as the anomaly of the 
sentences like John painted the door illegal (the latter may be attempted in the context where it is known that red is an illegal colour for doors).

Now that we explained where the resultative interpretation of the generalised conjunction comes from for c-states as the basis, we can sketch a path for an extension to SP-PP resultatives. This reading often comes about for verbs which have no expected results specified in the situation scheme, like run. It is customary to consider sentences like (43) to exemplify RC.

(43) John ran into the wood

Such PPs provide a point on a dimension to which the event is oriented. Locating some bearer of the event at this point creates a bounding state for the event, which otherwise lacks one. In other words, in case of PPRSP the bounding state is specified externally and there is no event coidentification involved. We use the bounding operation which however is not grammaticalized in English.

\section{Meaning and alternatives in Gricean contexts: A formalisa- tion}

Having made some points of the RSP-analysis by Rothstein more precise the next step is to formalise the reasoning intended to either generate the syntactic mapping of the situation scheme plus some additional communicative sense or to interpret a syntactic structure. Thus, what we propose amounts to an inferential theory of meaning.

\subsection{An abductive theory of meaning in context}

The aim of this section is to sketch an inferential theory of meaning in a Gricean context and to propose within it a mechanism which spells out the NOT-placeholder in the discussion of blocking in Gricean contexts in section 1.2.3. The inferential theory will help us to implement the desideratum of $\mathrm{G}$. Green of representing mild verb vagueness.

It is well known that a word can be used to refer to any number of things by the processes of metaphor and metonymy. It is not plausible that all of them must be checked in each context, where the listener has to arrive at a single interpretation of a syntactic structure as quickly as 
possible. A more plausible idea is that a context provides some material to compute a meaning of a verb whose general, context-independent meaning is underspecified. During this process some meanings must be discarded in a context, if they do not fit, preferably before they are computed. This is an old idea, which can be found already in Bréal (1887, p.141):

"It will be asked, how it is that these meanings do not thwart each other; but we must remember that each time the words are placed in surroundings which predetermine their import. We are not even troubled to suppress the other meanings of the word: these meanings do not exist for us, they do not cross the threshold of our consciousness. It is bound to be so, since happily the association of ideas is for most men based on essentials of things, and not on the sound".

The double-sided role of the context here is to provide a necessary amount of information for the interpretation on the one side and to help to weed out the implausible interpretations from this limited amount as early as possible on the other.

\subsection{What is abduction}

The whole task of integrating the contextual information into the meaning of the words and sentences and filtering out the implausible interpretations can be viewed as an instance of inference (association of ideas). Inference is defined in Genesereth and Nilsson (1987) as the process of obtaining conclusions from premises. Such a process is taken to consist of small inference steps each of which is justified by some inference rule. An inference rule consists of a set of sentence patterns called conditions in Genesereth and Nilsson (1987), and another set of patterns called conclusions. Whenever there are sentences which match the conditions of the rule we can infer sentences matching the conclusions. A rule is sound, if any set of conditions logically implies any set of conclusions derived from these conditions with the help of the rule. Deductive inference uses only sound rules, by definition. Sound forms of inference are not context-dependent, so, for instance, sound deductive rules are valid in any context, if valid in one particular context. Other forms of inference are not sound, yet are never- 
theless often claimed to be part and parcel of the human mental activity. The most promising candidate for the purposes of the theory of meaning among these is hypothetical inference. We adopt hypotheses to explain observations, and their adoption, considered as inference rule, is not a sound rule, since hypotheses may turn out to be false, given new observations. The biggest problem in modelling this mode of inference is how to specify the hypotheses.

Consider the case when some sentences match the patterns of the conclusions of a sound deductive rule, and we derive sentences which match the assumptions of the rule. Under this kind of inference we actually assume that we possibly deal with a more restricted set of models, than our observations alone tell us. Exactly what set this is is a matter of speculation, if no additional information is available, since there may be different sentences which match the pattern of conditions given the conclusions.

The main characteristics of hypothetical or assumption based reasoning is that we use ordinary logic to conduct it. We do not introduce new rules of inference in addition to the sound rules used in deductive inference, but change the use of these rules. Consider the case of the most conspicuous inference rule, modus ponens, (44).

$$
\text { (44) } \frac{p \quad p \rightarrow q}{q}
$$

Whenever we have $p$ and $p \rightarrow q$, the rule of modus ponens allows us to conclude $q$. Suppose we have $q$ and $p \rightarrow q$. Due to the soundness of the rule of modus ponens we know that $p$ implies $q$ relative to $p \rightarrow q$, so we may assume that we are dealing with the special case of the set of models satisfying $p$ to accommodate the observation that $q$ holds. We seem to be using modus ponens in the reverse direction. But assuming $p$ may be the wrong move, and the rule of modus ponens used in reverse direction is not a sound rule, hence the inference is not deductive. We simply consider $q$ to be an evidence that $p$ holds, because $p$ would imply $q$, given our knowledge that they are related, which is expressed by $p \rightarrow q$.

The notion has a flavour of explanation, since we seem to explain why $q$ holds, and the term has now become standard terminology for assumptions of this kind. As a technical notion it cannot be taken as an adequate explication of general ideas on what kind of thing an explanation is, though. 
Inference of this kind is more like guess-work, and some efforts must be undertaken to make it more justified or plausible. Different definitions of possible hypotheses as well as different criteria determining which of them may be used are possible. The fundamental criterion remains that the use of hypotheses should not lead to irresolvable conflicts. There are different uses of hypothetical reasoning. It can be used to explain observations, e.g. when we hypothesise that it has rained, if we come into the yard and see that the grass is wet. We can use hypothetical reasoning to predict situations, e.g. when we assume that the car we came to the office by is still at the place where we left it. We use it to contemplate alternatives, e.g. saying things like "If I were a carpenter, and you were a lady. ., you...". We could also use it to codify new knowledge given some observations, e.g. when we assume that all swans are white, having observed a number of white swans, but no swan of a different colour. The knowledge thus codified can be used in its turn for the tasks mentioned above.

The role of hypothetical reasoning in theory formation in natural sciences, e.g. in physics, is undisputed. Its usefulness as a mechanism of interpretation in natural-language based communication seems also to be acknowledged. The first extensive formal proposal to use a kind of hypothetical reasoning called abduction to model language comprehension was made in Charniak and McDermott (1985) to my knowledge. Abduction as one of the basic forms of reasoning alongside with deduction and induction first figured prominently in the works of Charles Saunders Peirce, who provided a short, essential characteristic of this inference form (Peirce, 1992, Lecture 2):

Still more convenient is the following conditional form of statement:

If $\mu$ were true, $\pi, \pi \prime, \pi \prime \prime$ would follow as miscellaneous consequences. But $\pi, \pi \prime, \pi \prime \prime$ are in fact true

$\therefore$ Provisionally, we may suppose that $\mu$ is true.

This kind of reasoning is often called adopting a hypothesis for the sake of its explanation of known facts. The explanation is the modus ponens 
If $\mu$ is true, $\pi, \pi \prime, \pi / \prime$ are true

$\mu$ is true

$\therefore \pi, \pi \prime, \pi / l$ are true

Recently a growth of interest in the use of abduction in linguistic theories could be noted, (e.g. Vasishth and Kruijiff (2001), Hobbs et al. (1993), McRoy and Hirst (1995), Meyer-Klabunde (1995), Norvig and Wilensky (1993)), probably as a consequence of the growing popularity of hypothetical reasoning over the years in the field of artificial intelligence, where it is used in plan recognition, diagnosis and common-sense reasoning in general, cf. Konolige $(1996)^{7}$. The theory we present here is also based on abduction.

\subsection{The tasks of an abductive theory of interpretation}

Consider what establishing the meaning of words and the communicative sense of sentences represented as propositional structures by hypothetical inference amounts to. Suppose we have a syntactic structure coded in some form which is easily convertible into propositions. Such propositions represent a very underspecified meaning of the sentence, the meaning which is computed in any context a sentence occurs in, i. e. it is context-independent. These context-independent underspecified semantic structures, which will be called semantic forms, stand in the relation of interpretation INT to more specialised semantic structures which are computed depending on the context. In a theory of semantic interpretation based on abductive reasoning INT is considered to be established by plausible inference. This inference can be described as assuming an appro-

\footnotetext{
${ }^{7}$ Difficulties in using abduction are considerable. Abduction is seldom tractable computationally (Eshghi, 1993), but some work on inductive logic programming shows that such results can be fruitful, and not detrimental, cf. Muggleton (1996). Kautz et al. (1995) note that "... abduction problems can be solved in polynomial time when the background Horn theory is represented by a set of characteristic models." They point out an interesting perspective: "... The fact that abduction is hard for clausal Horn theories, but easy when the same background theory is represented by a set of characteristic models, means that it may be difficult to generate the characteristic models of a given Horn theory: there may be exponentially many characteristic models, or even if there are few, they may be hard to find. None the less, it may be worth while to invest the effort to "compile" a useful Horn theory into its set of characteristic models, just in case the latter representation does indeed turn out to be of reasonable size. This is an example of "knowledge compilation"." These ideas may be relevant to the questions of semantics, too.
} 
priate available piece of conceptual knowledge for the sake of explaining these more abstract linguistic structures, semantic forms. A semantic form is thus deduced from the conceptual knowledge which we hypothetically adopt as relevant. If semantic forms are associated directly with some syntactic relations we have the induced relation of interpretation of these syntactic relations. If semantic forms are associated with words, words are interpreted. Of course, this must be made more explicit and formal.

A formalisation of abduction has two aspects. Talking about treatments of diagnosis in artificial intelligence, Raymond Reiter (Reiter, 1987) noted

Many non-monotonic inferences are abductive by nature, which is to say they provide plausible explanations for some states of affairs... The problem, of course, is that not just any explanation will do; it must, in some sense, be a "best" explanation... But if there is a best theory, there must be poor ones; so diagnostic reasoning really consists of two problems: (a) What is the space of possible theories that account for the given evidence? (b) What are the best theories in this space?

In this paper we will concentrate on the description of the space of available explanations which are relevant to the topic of the paper and on their structure and ignore the difficult question of how best explanations are chosen.

\subsection{Abductive frameworks after David Poole}

\subsubsection{Poole systems}

A simple formalisation of abduction for first order languages is as follows (Poole, 1988a): a subset $\mathrm{P}$ of ground instances ${ }^{8}$ of a set of some possible hypotheses $\Pi$ is an explanation for observation $\phi$, according to (45).

(45) $\Gamma \cup P$ explains $\phi$ if and only if

(i) $P \cup \Gamma \models \phi$

(ii) $P \cup \Gamma$ is consistent

The set of propositions $\Gamma$ represents our factual knowledge in the situation in which inference is done, $\phi$ is the observation to be explained, and $\mathrm{P}$ is the

\footnotetext{
${ }^{8}$ Ground instances are basically substitution instances of formulas in which all variables are replaced by constants.
} 
set of hypotheses available to us. Whenever hypotheses must be used each time they can be consistently used, we can speak of defaults. In this case we shall use the notation $\Delta$ for defaults. A formal theory with hypotheses $\Pi$ or defaults $\Delta$ and with the facts $\Gamma$ will be called abductive framework $A=(\Gamma, \Pi, \Delta)$. Note that defaults may, of course, be used as hypotheses, so there is no need to write them twice. It is only if some hypotheses are not defaults that they should be listed separately.

Let us consider an example. Suppose we have a theory which tells us that birds fly as a rule, but that ostriches definitely do not fly. Call this abductive framework $A_{b i r d}$. It has a set of defaults, $\Delta$, which contains the rule-like assumption that birds fly. The assumption is actually an open formula which gives rise to a hypothesis whenever all its variables are replaced by some constants. Such a substitution instance can be used as a hypothesis only if it is consistent, otherwise (45ii) is violated.

$$
\begin{aligned}
& A_{\text {birs }}=(\Gamma, \Delta) \\
& \Delta \quad=\{\operatorname{bird}(X) \rightarrow \operatorname{flies}(X)\}, \\
& \Gamma \quad=\left\{\begin{array}{l}
(\forall X)(\text { ostrich }(X) \rightarrow \operatorname{bird}(X)), \\
(\forall X)(\text { ostrich }(X) \rightarrow \neg \text { flies }(X)), \\
\text { ostrich }(\text { polly }), \\
\text { bird }(\text { tweety })
\end{array}\right\}
\end{aligned}
$$

This theory allows us to explain that tweety flies, but not that polly flies, because such an explanation would contradict the facts.

Using implications with open formulas as a formalisation of rules is sometimes unwieldy. There is a transformation which is equivalent with the original theory and which replaces such defaults and hypotheses by their names. The form of the names can be standardised to atomic predicates, and the names can serve as defaults or hypotheses then instead of the rules they stand for. Let $d\left(X_{1}, \ldots, X_{n}\right)$ be some default with the free variables $X_{1}, \ldots, X_{n}$. Let $d p r e d\left(X_{1}, \ldots, X_{n}\right)$ be a new $n$-place predicate which we want to use as the name of the default. We add it as a hypothesis or a default, add the implication $\left(\forall X_{1}, \ldots, X_{n}\right)\left(\operatorname{dpred}\left(X_{1}, \ldots, X_{n}\right) \rightarrow\right.$ $\left.d\left(X_{1}, \ldots, X_{n}\right)\right)$ as a new fact to $\Gamma$, and throw out the original default or hypothesis $d\left(X_{1}, \ldots, X_{n}\right)$. The new theory does not add any new deductions 
to the old one, as Poole (1988b) shown, but all the hypotheses and the defaults are now atomic predicates with as many free variables, as there are in the original defaults and no other parameters.

Consider a modification of (46) as an example of this transformation. The modification concerns the status of the rule for ostriches. Suppose we are not sure that all emus do not fly, although we expect them not to. Then $\operatorname{emu}(X) \rightarrow \neg f l i e s(X))$ is assigned the status of a default. Now we choose a new predicate to name this default and another one to name the original default. Then we have the standardised form of (46) and this modification is (47), where birdsfly $(X)$ and emusdontfly $(X)$ are the new atomic default predicates.

$$
\begin{aligned}
A_{\text {emus }} & =(\Gamma, \Delta) \\
\Delta \quad & \left\{\begin{array}{l}
\text { birdsfly }(X) \\
\text { emusdontfly }(X)
\end{array}\right\} \\
\Gamma \quad & \left\{\begin{array}{l}
(\forall X)(\text { emu }(X) \rightarrow \operatorname{bird}(X)), \\
(\forall X)(\text { birdsfly }(X) \rightarrow(\text { bird }(X) \rightarrow \text { flies }(X))), \\
(\forall X)(\text { emusdontfly }(X) \rightarrow(\text { emu }(X) \rightarrow \neg \text { flies }(X))) \\
\text { emu }(\text { polly }), \\
\text { bird }(\text { tweety })
\end{array}\right\} .
\end{aligned}
$$

Now we can explain both flies(polly) and $\neg$ flies(polly). Since the two explanations are contradictory, we would like to have some criteria which could decide which explanation is to be preferred. We could use some notion of specificity, for instance. Since emus are birds, the default about emus is more specific, than the default about birds. The latter covers normally emus, too, and if we want the more specific default to be used at all, higher specificity should enforce this.

Consider another feature of abductive frameworks. Since we reason in ordinary logic we could use contrapositives to the defaults. Suppose we know that some individual tweety does not fly, i. e. $\neg$ flies(tweety). Then, using contrapositives we could conclude that it is not a bird and hence not an emu. This could turn out to be wrong and the more specific default on non-flying emus does not apply. We could, however, block the 
contrapositives of the most general defaults if we wish by introducing the notion of constraints.

A constraint is a formula which can be used to reject an explanation, but which cannot be used in deduction, e. g. to augment explanations. The definition of an explanation in an abductive framework with constraints, defaults and hypotheses is given in (48).

(48) $\Gamma \cup P \cup D$ explains $\phi$ if and only if

(i) $\Gamma \cup P \cup D \models \phi$

(ii) $\Gamma \cup P \cup D \cup C$ is consistent

where $D, P$ are ground instances of formulas from the set of defaults $\Delta$ and hypotheses $\Pi$, and $C$ are ground instances of the set of constraints $\mathcal{C}$. An abductive framework is then a quadruple

$$
A=<\Gamma, \Pi, \Delta, \mathcal{C}>
$$

Now we may add a constraint to the effect that the contrapositives of at least most general defaults cannot be used.

$$
\neg \operatorname{flies}(X) \rightarrow \neg \text { birdsfly }(X)
$$

Thus, though we cannot use the constraint to derive conclusions, we use it to control the possible applications of defaults. A consequences of such a use of constraints is that defaults cease to be modular and independent of each other. If we are not sure that contrapositives are useful, we could to use blocking constraints for all the defaults.

\subsubsection{NAF and the selection mechanism in Gricean contexts}

Consider again the case $\neg$ flies (tweety). We cannot infer that tweety is not a bird in (47), given that the contrapositives are blocked. What can we assume? We could assume that tweety is an emu, of course, but it could be a non-bird, too. Suppose we also know that it is a bird. Moreover, we know our emus personally, and tweety does not seem to be one of them. It seems to be plausible to conclude that tweety is not an emu. The relevant rule could be "if a bird doesn't fly, but it cannot be proved that it is an emu, assume that it is not an emu".

In logic programming (Lifschitz (1996))(Nilsson and Maluszyński (1990)) such a rule is called Negation As Failure or $N A F$ for short. We can model 
this kind of rule in abductive frameworks. Thus, (49) contains a formalisation corresponding to the rule in the paragraph above.

$$
\begin{aligned}
& A_{\text {emus }}=(\Gamma, \Delta, C) \\
& \Delta \quad=\left\{\begin{array}{l}
\text { birdsfly }(X) \\
\text { emusdontfly }(X) \\
\sim \text { emu }(X)
\end{array}\right\} \\
& \Gamma \quad=\left\{\begin{array}{l}
(\forall X)(\text { emu }(X) \rightarrow \operatorname{bird}(X)), \\
(\forall X)(\text { birdsfly }(X) \rightarrow(\text { bird }(X) \rightarrow \text { flies }(X))), \\
(\forall X)(\text { emusdontfly }(X) \rightarrow(\text { emu }(X) \rightarrow \neg f l i e s(X))) \\
(\forall X)(\sim \text { emu }(X) \& \text { bird }(X) \& \neg \text { flies }(X) \rightarrow \neg \text { emu }(X)) \\
\text { emu }(\text { polly }), \\
\text { bird }(\text { tweety }) \\
\text { emu }(X) \& \sim \text { emu }(X) \rightarrow \perp \\
\neg f l i e s(X) \& \text { birdsfly }(X) \rightarrow \perp
\end{array}\right\} \\
& C \quad
\end{aligned}
$$

The predicate $\sim e m u(X)$ in this framework is the NAF-default. It is a contrary to $\mathrm{emu}(X)$ and takes care of the negation of the latter. Basically, to model NAF we introduce a unique name $\sim P$ for each predicate $P$ the negation of which should be automatically assumed, if the predicate cannot be proved. We also add a corresponding default which states exactly this: assume the negation of the predicate. The default (resp. the name of the rule in the standardised form) cannot co-occur with the predicate. This is fixed by a constraint. Being a default, it should be used unless nothing contradicts it. The second constraint blocks the contrapositive of the general default, as described in the previous section. This constraint is of a different kind than the NAF-constraint.

The kind of defaults which model the NAF-rule may be put to a more general use by using consequents in the rule which differ from the negation of the predicate. Suppose we want to decide what to do if a bird does not fly. We might continue assuming it is not an emu. But what of it? We would welcome an advice in this case, e. g. perhaps we should ask John. This can be managed by an additional fact (rule) in (50) which adds dependent information for the case we cannot prove a non-flying bird to 
be an emu.

$(50)(\forall X)(\sim \operatorname{emu}(X) \& \operatorname{bird}(X) \& \neg f \operatorname{fies}(X) \rightarrow \operatorname{ask}(X, j o h n))$

The device of introducing a hypothetical contrary to a fact can be extended to defaults (instead of fact predicates). Suppose there is a contrary default for each standard default name. Such a pair of conjugate defaults gives us mutually exclusive alternatives. It can therefore be used to implement the NOT abbreviation we used in characterising Gricean contexts. Hence, this can be the mechanism underlying mutual blocking of some mapping rules in the general case and in case of event co-identification in particular. Obviously, such a device should be restricted to a context.

\subsection{The description of the task continued}

\subsubsection{From abduction to interpretation}

To put the basic ideas of abductive reasoning into use in a theory of interpretation, we postulate a semantic level of representation the formulas of which constitute a kind of semantic form of a sentence, representing the interpretable syntactic relations and properties, including the reference to the lexical knowledge invoked by the sentence. These formulas are treated as evidence to be explained in the formal sense we defined above by more specific contextual meanings. The explanations draw on the resources which are available in the context of interpretation.

These considerations invite the following three components of the theory of INT: semantic forms associated with the syntax, contextually specified knowledge, and rules that relate the knowledge and the observations. The task of the theory of interpretation in case of a sentence $\phi$ may then be formally described as finding an abductive framework $A$ such that $A=$ $(\Gamma, \Pi, \Delta)$ and for some $P, D$

$$
(I N T) \quad \Gamma \cup P \cup D \models_{c} S F(\phi)
$$

In this formula, $D$ and $P$ are subsets of ground instances of formulas in $\Pi, \Delta$ respectively, $c$ a context and $S F(\phi)$ is the semantic form of $\phi$. We will call $c$ the context of interpretation.

The observations depend on the structure of the sentence. Since syntactic structures are specified recursively, semantic forms must be recursively 
specifiable, too. Moreover, the image of the syntax under INT should, in principle, determine the truth conditions for $\phi$ in the context of interpretation. These are important questions. However, for the limited purposes of the present paper, we will proceed on a case-by case basis, making specific decisions whenever the need arises and avoiding the generalities of such a theory.

First we integrate contexts in the theory of abductive interpretation. To simplify things we introduce only four kinds of context:

1. The context of the situation

2. The context of the verbal projection

3. The context of some grammatical relations

4. The context of the generalised conjunction.

Each of these contexts introduces some discourse referents in the manner of Kamp and Reyle (1993) and an abductive framework as its inferential resources: facts, defaults, hypotheses and constraints. The next section discusses some properties of the contexts.

\subsubsection{Contexts}

A very useful notion of context was introduced in the paper McCarthy and Buvač (1998). Though not a complete formal theory of contexts, it is a collection of interesting proposals and illustrations. Other notions of context might turn out to be more appropriate, of course.

A context in this theory is a formal object, hence an object, and can thus be a value of a first-order variable. Since any statement is made in a context, it should be relativised to something like context $(x) \rightarrow A$, where $A$ is the formula which makes a statement, and context $(x)$ fixes the context. A context is also a generalisation of the notion of a collection of assumptions which build up a kind of an axiomatic base of the context. One important difference between an explicit collection of assumptions and a context, which suggests using contexts as objects instead of mere sets of assumptions, is that a context could contain a number of assumptions not known to us, i. e. we cold have only a partial knowledge of the context. 
To state that an assertion $A\left(a_{1}, \ldots, a_{n}\right)$ is true in some context McCarthy and Buvač propose to use the predicate $i s t(c, p)$, which means proposition $p$ is true in context $c$. Note that this statement is itself only true in some context, i. e. $i s t(c l, i s t(c, p))$. Similarly, the latter formula is only true in a context. This creates an infinite regress, but the latter is harmless, as the authors indicate. We call the current most general context in which which we reason outer context, following McCarthy and Buvač.

A useful piece of notation to indicate that our reasoning takes place in a context is to prefix all statements $A\left(x_{1}, \ldots, x_{n}\right)$ made in a context by the name of this context, i. e. to write $c: A\left(x_{1}, \ldots, x_{n}\right)$ e. g.

$$
\text { writing-a-letter : "I fell asleep". }
$$

Another way to indicate this is to conditionalise the statement on the context assumption, e. g. to write

$$
\text { context }(\text { writing }-a-\text { letter }) \rightarrow \text { "I fell asleep". }
$$

and use the context assumptions as assumptions in the sense of natural deduction, i. e. after assuming context(writing-a-letter), and deriving something, we conditionalise on the context and discharge this assumption.

Inference in a given context is done by entering a context, performing the inference, and exiting it, concluding that some fact is true in a context. Entering a context $c t$ and asserting $p$ in this context is notated using the same : prefix, cf. (51).

(51) $c: p$

If $c^{\prime}: i s t(c, p)$, and we have entered context $c$ and inferred $q$ from $p$ in $c$, we can leave this context and obtain $c^{\prime}:$ ist $(c, q)$.

To relate context and situations we associate a context with a situation, $c t(s)$. Using this device as a Skolem function we may call this context the context of the situation or situation context. To model abductive reasoning in context we associate an abductive framework with a context, writing $A_{c}$. An abductive framework associated with a situation context for $s$ is then $A_{c t(s)}$.

Doing abductive reasoning relative to contexts poses some problems. Abductive reasoning in a context $c$ sometimes allows us only to obtain an 
explanation for some proposition but not its proof. It is then necessary to have the relation of explanation in a context, e. g. $c^{\prime}: \operatorname{expl}(c, p)$. If we change contexts, the explanations may become invalid, i. e. it may be $c^{\prime}: \neg A$ for some $A$ used in the explanation for $p$ in $c$. Then we either cannot use $p$ or should look for another explanation which does not involve $A$. Such an explanation may be obtainable in some other context. Since we are not developing a full theory of abductive explanation in a context, we will simplify matters and import not only statements inferred in $c$, but also facts, defaults, hypotheses and constraints. In the paper this amounts to taking unions of the components of abductive frameworks discussed in the section 3.5.1.

It may become necessary to import the inferences made in context $c$ into its outer context. To do this we should provide for those predicates $P(x)$ which we want to import from $c$ to $c^{\prime}$ their rendering in $c^{\prime}$. A solution wold be to let $c^{\prime}$ contain facts of the form $c^{\prime}: \operatorname{expl}(c, P(x)) \rightarrow P^{\prime}(x, \ldots)$. But unifying the contexts instead of jumping from an inner context to an outer context keeps things simple in this paper. It is sufficient to have $c^{\prime}: P(x) \rightarrow P^{\prime}(x, \ldots)$, since we can verify in $c^{\prime}$ whether $P(x)$ is explainable.

Four kinds of contexts seem to be sufficient to model INT here:

- The context of the situation

The context of situation $s, c t(s)$ provides a situation template. It contains some information about characteristic properties of a situation and its structure in general, e. g. (52):

$$
A_{c t_{s}}=\left(\Gamma_{c t_{s}}, \Delta_{c t_{s}}, \Pi_{c t_{s}}, C_{c t_{s}}\right)
$$

The facts, defaults, etc about a situation may include properties of predicates involves in a situation frame, e. g.

$$
\Gamma_{c t_{s}}=\left\{\begin{array}{l}
\operatorname{situation}(s, e 1, e 2, t, \operatorname{tr}(s), \tau(s)) \rightarrow \ldots \\
\operatorname{proc}(e 1, s) \rightarrow \ldots \\
\operatorname{init}(e 1, e 2) \rightarrow \ldots \\
\ldots \text { etc. }
\end{array}\right\}
$$

Situation contexts will serve as outer contexts in the paper. The semantic form of the sentence is the observation to be explained in $c t(s)$. 
Leaving out the problems of quantification and assuming only existential quantification for the rest of the paper, the construction of the semantic form of the sentence in a language of first order logic may follow any standard compositional procedure. We need not be explicit and so the intended semantic forms will be simply indicated.

- The context of a verb

This context is intended to provide the contribution of the verb to the explanation of SF. It contains a situation scheme associated with the verb.

- The context of a grammatical relation

Though it is not obvious that interpreting syntactic relations by semantic relation is necessary, it was usual, e. g., in Montague grammar and it seems to be the easiest way to implement neo-davidsonian semantics. All interpretable syntactic relations within the maximal VP are registered and interpreted here.

The contexts of grammatical relations introduce both the facts describing which syntactic relation is to be interpreted, and the associated observations as context-independent predicates which are to be explained. This move generalises linguistic views on thematic relations (in the sense close to semantic roles).

- The context of an adjunct generalised conjunction

The context of the generalised conjunction sums two relevant eventualities as an interpretation. The summing operation is a default interpretation of syntactic adjuncts which are syntactically typed as eventualities. The sum is the new discourse referent in the context.

\subsection{Computing a [+bounded] semantic form in Russian}

Let us consider a derivation of a semantic form in Russian as an example. In doing this we shall introduce some notions relevant to the treatment of c-states in Russian.

The sentence (53) has a perfective verb, which, as will be proposed in section 4.4, exemplifies a bounded evientuality predicate. 
(53)

Masha razbudila Pet'u

Masha wake-perf-past Peter

Masha woke up/has woken up Peter

Assume that the relevant piece of the syntactic structure of the sentence is something like (54).

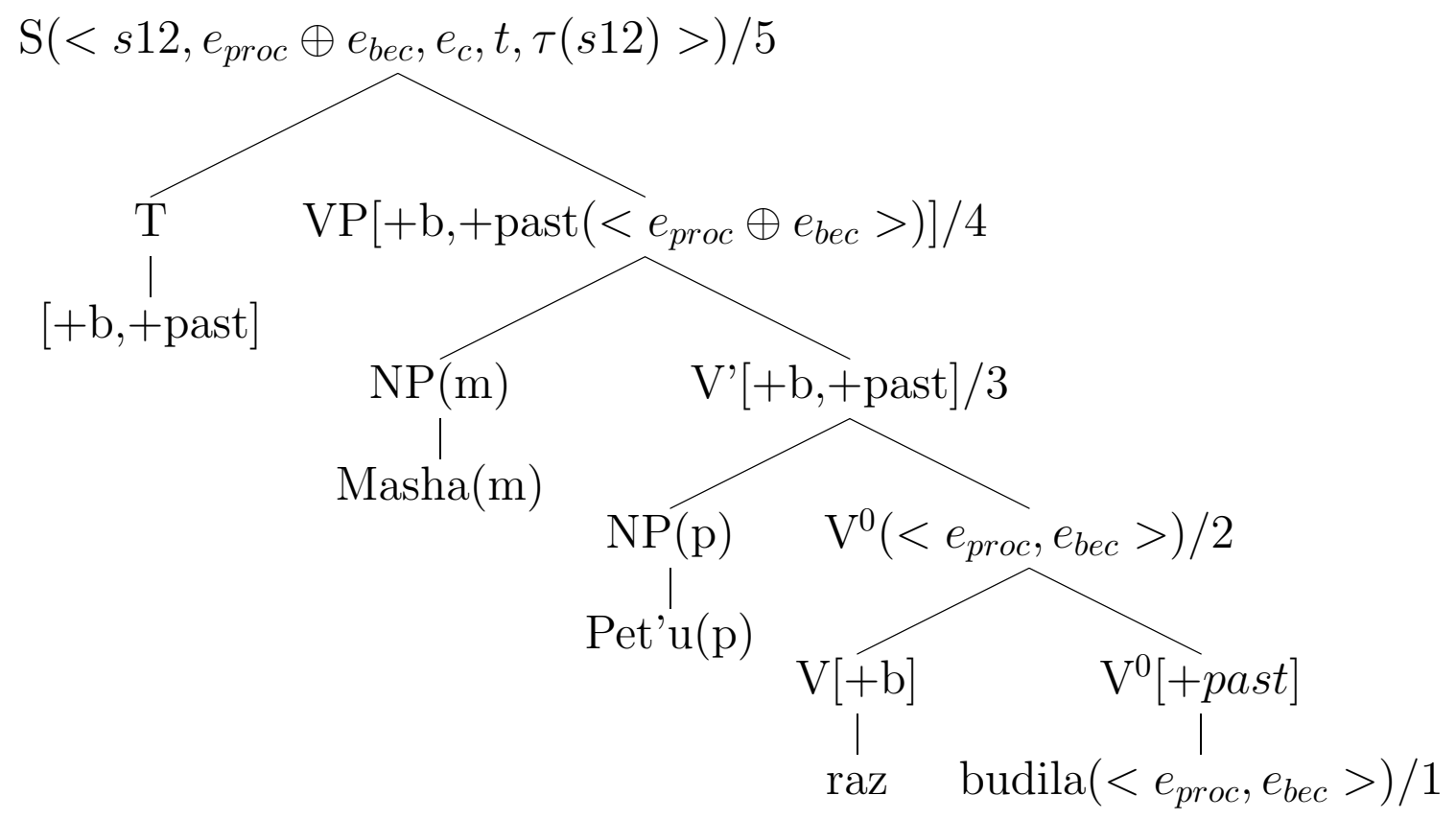

We shall make a number of simplifications. The discourse referents which are indicated in parenthesis are constants and not variables. No distinction between syntactic and semantic indices is made. The NP constituents are assumed to have been explained, with explanations given as terminal labels. Since we can form the sum of any two events any time, we need not state this explicitly; starting with the maximal verbal projection we have the sum of the two events specified by the verb as the discourse referent. The syntactic feature $[+b($ ounded $)]$ must be interpreted, and is always interpreted by the predicate bounds, as we shall see later.

\subsubsection{The semantic form}

The semantic form of an expression is simply a formal propositional counterpart in a first order language of the syntactic relations and lexical nodes in the sentence which must be interpreted. The semantic form of (53) is given in (55). 
(55) budi $\left(e_{\text {proc }} \oplus e_{\text {bec }}\right) \& \operatorname{argsubjec}\left(m, e_{\text {proc }}\right) \& \operatorname{argobject}\left(p, e_{\text {bec }}\right)$

$\&$ bounds $\left(e_{\text {proc }} \oplus e_{\text {bec }}, e_{c}\right) \& \operatorname{proc}\left(e_{\text {proc }}, s 12\right)$

$\&$ consstate $\left(e_{\text {proc }}, e_{c}\right) \&$ masha $(m) \& \operatorname{peter}(p)$

The syntactic tree contains references to the contexts in the form of numbers preceded by a slash. In describing the contexts introduced at the node we shall list the contribution of the node to the semantic form, too, thus indicating the origin of the parts of this expression. We start constructing the context of the sentence by creating a context of the situation. In doing this we use the corresponding abductive frame of the situation and list the expressions to be explained using the resources of this or other contexts, if there are any.

3.6.2 The context of the sentence: $/ 5$

$$
\begin{aligned}
& A_{(c t(s 12))}=
\end{aligned}
$$

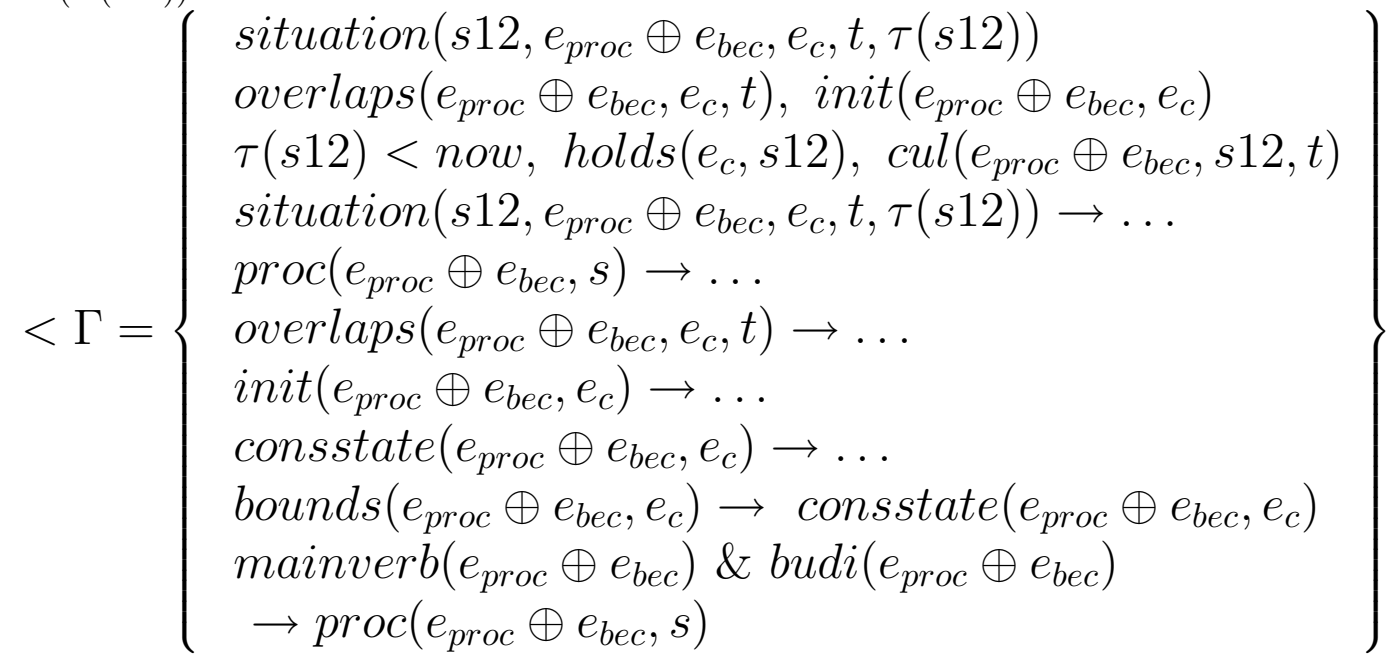

$$
\begin{aligned}
& \Delta=\emptyset \\
& \Pi=\left\{\text { mainverb }\left(e_{\text {proc }} \oplus e_{\text {bec }}\right) \ldots\right\} \\
& C=\emptyset> \\
& \text { explain : proc }\left(e_{\text {proc }} \oplus e_{\text {bec }}, s\right) \text {, consstate }\left(e \oplus e_{\text {bec }}, e_{c}\right) \text {, budi }\left(e_{\text {proc }} \oplus e_{\text {bec }}\right)
\end{aligned}
$$

A proper formal implementation of the construction algorithm would use formulas with open variables as defaults and hypotheses, and quantified formulas as facts. But since discourse referents must instantiate variables in the formulas before computing explanations, we skip the algorithm and simply use the necessary instantiations. The facts of $\Gamma_{(c t(s 12))}$ express two 
things: they state what is not in need of explanations in our example, and provide some fixed properties of some basic predicates. We have to explain what the process of the situation is, what its consequent state is, and why the stem budi was used. The form consstate $\left(e_{\text {proc }} \oplus e_{b e c}, e_{c}\right)$ needs to be explained because it is specified as holding in the situation.

In principle, the rules which are defaults from the perspective of the speaker should yield hypotheses from the perspective of the hearer. But it is only natural that defaults may always be used as hypotheses.

\subsubsection{The context of the subject-relation: /4}

The context of the syntactic subject of the sentence relation makes two contributions. It contributes a fact that it holds for a certain discourse referent and it provides an evidence which has to be explained. The first provision is necessary because, e. g. dummy subjects do not provide a standard interpretation, as a rule.

$$
\begin{gathered}
A_{(c t(g f s u b j))}=<\Gamma=\left\{\begin{array}{c}
\text { gfsubject }\left(m, e_{\text {proc }}\right) \\
\text { agentsubj }\left(m, e_{\text {proc }}\right) \text { \& agent }\left(m, e_{\text {proc }}\right) \\
\rightarrow \operatorname{argsubj}\left(m, e_{\text {proc }}\right)
\end{array}\right\} \\
\left.\begin{array}{c}
\Delta=\left\{\operatorname{agentsubj}\left(m, e_{\text {proc }}\right)\right.
\end{array}\right\} \\
\Pi=\emptyset \\
C=\emptyset> \\
\hline \text { explain : argsubj }\left(m, e_{\text {proc }}\right)
\end{gathered}
$$

3.6.4 The context of the object-relation: /3

The context of the syntactic object of the sentence relation makes two parallel contributions. This time the fact of its holding is necessary to distinguish cases of implicit objects.

$$
\begin{aligned}
& A_{(c t(g f o b j))}=<\Gamma=\left\{\begin{array}{l}
\text { gfobject }\left(p, e_{b e c}\right) \\
\text { themeobj }\left(p, e_{b e c}\right) \& \text { theme }\left(p, e_{b e c}\right) \\
\rightarrow \operatorname{argobject}\left(p, e_{b e c}\right)
\end{array}\right\} \\
& \Delta=\left\{\text { themeobj }\left(p, e_{b e c}\right)\right\} \\
& \Pi=\emptyset \\
& C=\emptyset> \\
& \text { explain : argobject }\left(p, e_{b e c}\right)
\end{aligned}
$$


3.6.5 The context of the $\mathrm{V}^{0}$-adjunct relation: / 2

The contribution of the verbal prefix context is in general much more complicated, than shown here, as the discussion in section 4.4 will show. This representation is provisional.

$$
\begin{aligned}
& \left.A_{(c t(r a z))}=<\Gamma=\left\{g f \_v \_a d j u n c t\left(e_{c}, e_{\text {proc }} \oplus e_{b e c}, s\right)\right)\right\} \\
& \Delta=\left\{\operatorname{cstate}\left(e_{\text {proc }}, e_{c}\right) \rightarrow \text { bounds }\left(e_{c}, e_{\text {proc }}\right)\right\} \\
& \Pi=\emptyset \\
& C=\emptyset>
\end{aligned}
$$

3.6.6 The context of the verb: /1

$$
\begin{aligned}
& A_{(c t(b u d i))}=\Gamma=\left\{\begin{array}{l}
\text { wake }\left(e_{\text {proc }} \oplus e_{\text {bec }}, s\right) \\
\text { wake }\left(e_{\text {proc }} \oplus e_{\text {bec }}\right) \rightarrow \operatorname{budi}\left(e_{\text {proc }} \oplus e_{b e c}\right) \\
\text { waker }\left(m, e_{\text {proc }}\right), \text { sleeper }\left(p, e_{\text {bec }}\right), \\
\text { waking }\left(e_{\text {proc }}\right), \text { awaking }\left(e_{\text {bec }}\right) \\
\text { waker }\left(m, e_{\text {proc }}\right) \& \operatorname{sleeper}\left(p, e_{\text {bec }}\right) \& \\
\text { expected_result_awaking }\left(e_{\text {proc }}, e_{c}\right) \\
\rightarrow \text { awake }\left(e_{c}\right) \& \exp \left(p, e_{c}\right) \\
\text { waker }\left(m, e_{\text {proc }}\right) \rightarrow \text { agent }\left(m, e_{\text {proc }}\right) \\
\text { expected_result_awaking }\left(e_{\text {proc }}, e_{c}\right) \\
\rightarrow \text { cstate }\left(e_{\text {proc }}, e_{c}\right) \\
\text { sleepertheme }\left(p, e_{b e c}\right) \& \operatorname{sleeper}\left(p, e_{b e c}\right) \\
\rightarrow \text { theme }\left(p, e_{b e c}\right)
\end{array}\right\} \\
& \Delta=\left\{\text { sleepertheme }\left(p, e_{\text {bec }}\right)\right\} \\
& \Pi=\left\{\text { expectedresult_awaking }\left(e_{\text {proc }}, e_{c}\right)\right\} \\
& C=\emptyset>
\end{aligned}
$$

We explain consstate $\left(e_{\text {proc }}, e_{c}\right)$ by expected_result_awaking $\left(e_{\text {proc }}, e_{c}\right)$. This account of the interpretation of $[+b$ (ound)] in Russian is simplified in two ways. First, the operation of event bounding does not figure by itself, but is rather associated with the prefix. This is not the final word on it, since no interaction between the operation and the prefixes is specified. Second, there is as yet no visible potential point at which Russian would conflict with English. 


\section{The core theory of the RSP blocking hypothesis for Russian}

\subsection{Disjunctive defaults via NAF}

Two basic structuring principles were assumed to govern the construction of rule sets in Gricean contexts in section 1.2.3. we repeat them here in a more technical formulation.

- Uniqueness of realization (UR)

Two different argument relations cannot be explained with the help of one and the same semantic relation in the same context.

- Uniqueness of interpretation (UI)

An argument relation cannot be explained by two different semantic relations in the same context.

In this formulation semantic relations used in the explanation are actually parts of the defaults. A more accurate statement would use standard default names instead.

What we suggest here is that these are not simply principles which make sense, but are consequences of a simple inferential mechanism which is used to structure rule sets. The mechanism is the one of handling alternatives and is based on the possibility to use conjugate defaults noted at the end in section 3.4.2. The idea is well known in linguistics under the name of disjunctive rule ordering. Informally, two rules are disjunctively ordered, if exactly one of them may be used in a given context. In terms of abductive frameworks, two rules are disjunctively ordered, if they are named by conjugate defaults. Note however that since we generalised the NAF rule there is no restriction to one possible conjugate rule to a given default. Any of these rules may use the conjugate default.

Consider the simplest case of one step explanation. UR may be rendered by a pair of conjugate defaults schematically represented as (61). 


$$
\begin{gathered}
A=<\Gamma=\left\{\begin{array}{l}
\text { default \&some_relation } \rightarrow \text { argrelation } 1 \\
\sim \text { default \&some_relation } \rightarrow \text { argrelation } 2
\end{array}\right\} \\
\Delta=\left\{\begin{array}{l}
\text { default } \\
\sim \text { default }
\end{array}\right\} \\
\Pi=\emptyset \\
C=\{\text { default \& } \sim \text { default } \rightarrow \perp\}>
\end{gathered}
$$

\section{explain :}

As for UI we require that any two alternative explanations in the linking rules are conjugate defaults. The scheme for one-step explanations is (62).

$$
\begin{aligned}
A=<\Gamma=\left\{\begin{array}{l}
\text { default \& relation } 1 \rightarrow \text { argrelation } \\
\sim \text { default \& relation } 2 \rightarrow \text { argrelation }
\end{array}\right\} \\
\Delta=\left\{\begin{array}{l}
\text { default } \\
\sim \text { default }
\end{array}\right\} \\
\Pi=\emptyset \\
C=\{\text { default \& } \sim \text { default } \rightarrow \perp\}>
\end{aligned}
$$

$$
\text { explain: }
$$

To cover the general case we require that any default used in either patterning or in linking rules is disjunctive. Now we have all the components of a theory of Russian bounding states.

\subsection{The basic treatment}

4.3 The context of $\oplus$ and the interpretation of RSP in English: painting something red

It is now possible to supply the formal details of the treatment of RSP in English sketched in section 2.8. The syntax we assume for RSP is basically as in (64). The idea that secondary predication involves a predicative phrase is due to Bowers (1993). The conjugate defaults are left out. They become relevant in section 4.3.3.

(63) John painted the door red 
(64)

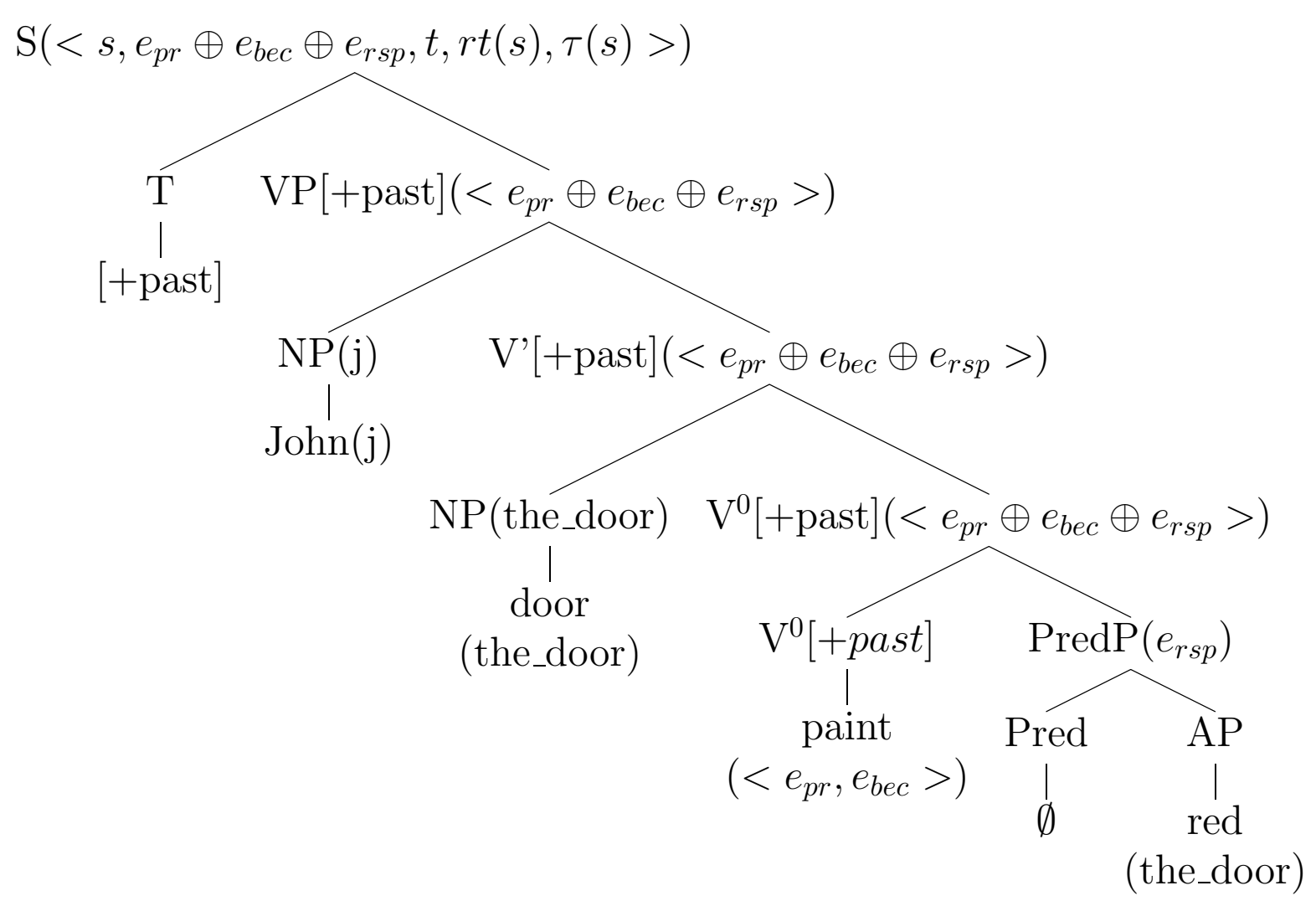

Note that there is no feature $[+\mathrm{b}$ (ound) $]$ on the secondary predicate phrase. It is a V0-adjunct like Russian prefixes. However, secondary predicates are a general device and are not associated with any particular semantics, hence there is no need to provide an interpretable syntactic feature.

We are interested in the relation associated by default with the verbal adjunct relation relating a verb and a predicative phrase. Let us call this relation $g f_{-} \operatorname{PrP}$ _inf. The interpretation of the conjunction is based on the free choice of the conjoint eventuality among those provided by the verb. It is relative to the context of the syntactic relation, of course, since we would not like to assume the part-of relation $\leq$ for generalised conjunction in general. Also, recall that we can treat any sum of two or more eventualities as an eventuality. 


$$
\begin{aligned}
A_{\left(c t\left(\oplus \operatorname{Pr} P \_i n f\right)\right)} & <\Gamma=\left\{\begin{array}{l}
g f_{\_} P r P \_i n f\left(e_{p r} \oplus e_{b e c}, e_{r s p}\right), \\
e_{\oplus}=e_{p r} \oplus e_{b e c} \oplus e_{r e s}, \\
r e d\left(e_{r e s}\right), \exp \left(e_{r e s}, \text { the_door }\right)
\end{array}\right\} \\
\Delta & =\emptyset \\
\Pi & =\left\{e_{b} \leq e_{r e s}, e_{p r} \leq e_{r e s}, e \leq e_{r e s},\right\} \\
C & =\emptyset>
\end{aligned}
$$

explain : we skip this part, e. g. explaining red or exp

In formalising the inference steps by an abductive framework we should always keep in mind that the inference of the co-identification is strictly speaking only possible in the context of the secondary predication, but can be exported into the context of the situation. To show this dependence we write $\operatorname{ct}(s)$ :.

$$
\begin{aligned}
& A_{\left(c t\left(\oplus c_{\_} s t a t e\right)\right)}=<\Gamma=\left\{\begin{array}{l}
\left.c t(s): \text { makecstate } 1\left(e, e_{b}, e_{r e s}\right), e_{\oplus}\right) \\
\& \text { cstate }\left(e, e_{b}\right) \\
\& e_{b} \leq e_{r e s} \& e_{\oplus}=e \oplus e_{\text {res }} \\
\rightarrow c_{-} \text {state }\left(e, e_{\text {res }}\right) \\
\left.c t(s): \text { makecstate } 2\left(e, e_{b}, e_{\text {res }}\right), e_{\oplus}\right) \\
\& \text { cstate }\left(e, e_{b}\right) \& c_{-} \text {state }\left(e, e_{\text {res }}\right) \& e_{b} \leq e_{\text {res }} \\
\rightarrow e_{\text {res }} \leq e_{b}
\end{array}\right\} \\
& \Delta=\left\{\begin{array}{l}
\text { makecstate } 1\left(e, e_{b}, e_{r e s}, e_{\oplus}\right) \\
\text { makecstate } 2\left(e, e_{b}, e_{r e s}, e_{\oplus}\right)
\end{array}\right\} \\
& \Pi=\emptyset \\
& C=\emptyset>
\end{aligned}
$$

\section{explain :}

Now, coidentify $\left(e_{b}, e_{r e s}\right)$ is derivable from $e_{b} \leq e_{\text {res }} \& e_{b} \leq e_{\text {res }}$. The complete representation of the situation is given in (67), omitting some details. Note that because of the co-identification and the law of the idempotence $e_{\oplus}$ is equal to $e_{-} r s p$. 


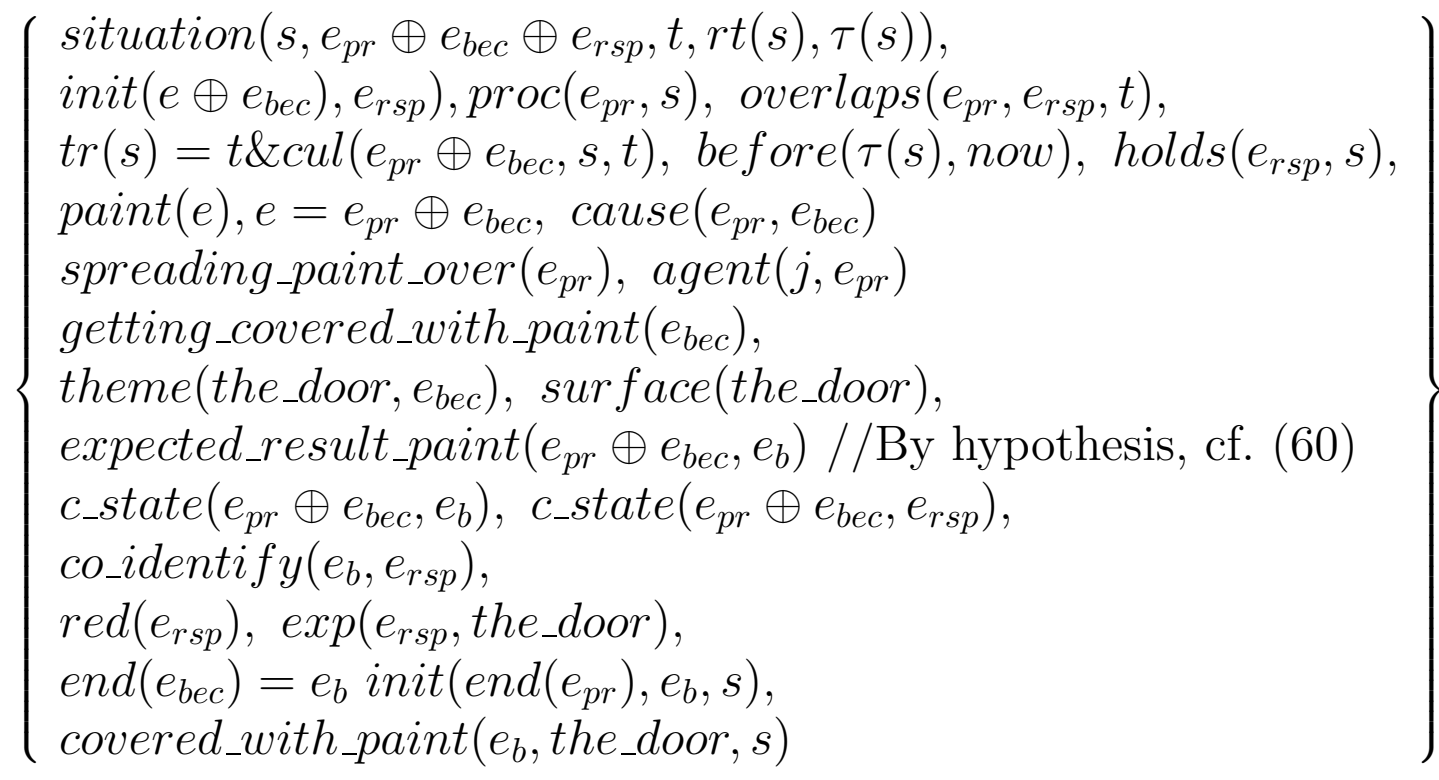

The next step is to show where the interpretation mechanisms sketched for Russian in section 3.6 come into conflict with the strategy of English, excluding it in Russian. This will be done in three steps. First, a grammaticalization of the event bounding operation in Russian will be sketched. Second, we shall show that Russian perfective verbs refer to bounding states as their primary characteristics, and not to r-states, like the English perfect forms. And lastly we will show where the two interpretation patterns conflict.

\subsubsection{Russian c-states and event bounding}

We described event bounding as a cognitive patterning operation which is used to systematically organise conceptual information to be mapped to syntactic structures. The fundamental point of the theory is that cstates in Rothstein's sense of expected results are always bounding states on bounding dimensions in Russian. We now suggest that Russian has a syntactic device which is systematically associated with event bounding. Hence, if a c-state is asserted to hold in a situation, the device expressing that there is a bounding state must be used as the preferred one.

Some knowledge of Russian data is necessary to see this point. The following short sketch of the Russian data draws on Wade (1992) and Švedova (1970). 
It is a well-known fact in Russian linguistics that the whole set of Russian verbs is fairly accurately divided into two halves by a simple test ${ }^{9}$. If the verb may co-occur with verbs denoting either the beginning, the end or the continuation of a process it belongs to the class of verbs of imperfective aspect, if it may not, it is said to belong to the class of verbs of the perfective aspect, cf (68), where -imp and -perf denote imperfective and perfective classes of verbs, respectively. Perfective verbs are not used in the present tense.

(68) a. *Ja načinaju narisovat'

J start-imp draw-perf

*I start to have drawn

b. Ja načinaju risovat'

J start-imp draw-imp

I start to draw

(Peškovskij, 1956, p. 108) notes that aspectual properties of infinitival forms remain the same for all other forms.

It is also a well-known fact that the the opposition perfective vs. imperfective aspect is systematic, with many verbs building aspectual pairs, though there are verbs with only one aspect. Two terms of such an aspectual opposition may be related by prefixation, suffixation or internal morphological modification, in some instances by stress, and may also be derived from different roots. Since the characteristics of the partition do not depend on the formal expression of the opposition, we shall use the term perfective tag to denote the abstract perfectivisation operator.

Wade notes that though the perfective tags tend to be semantically neutral, that is they change the aspect of the verb, but not its meaning, many prefixes can impart additional meaning which may differ for different verbs. Wade describes the basic, aspectual meaning of perfectivising prefixes as follows:

The perfective focuses on the completion of a single action in the past or future. Usually a result is implied... Often the perfective denotes the culmination of a process.

\footnotetext{
${ }^{9}$ There are some ambiguous verbs; cf. Wade (1992) who calls them bi-aspectual.
} 
The neutral meaning of a tag usually implies an expected result. Additional meanings of this tag may imply different results. Consider the prefix raz/ras- as an illustration of Wade's data ${ }^{10}$. The comments after '//' are intended to show the common structure of the different uses:

i. Separation, dispersal, disintegration, distribution:

razdvinut' zanaveski

to part the curtains // be moving until they are apart

razmestit' rakety

to deploy missiles // be assigning a place until each has one

raspilit' stvol

to saw up a tree trunk // be sawing until it is in parts

ii. Reversal of an action

razv'azat'

to untie // be manipulating some knotted thing until it is apart

razdumat'

to change one's mind // be thinking until the original intention is adopted again

Note that the concept of separation is defined relative to other lexical information of the verb; similarly for the reversal of the action. That is, the meaning of the prefix is rather general and should be further specified depending on the verb it applies to.

Švedova (1970) lists some more uses, the neutral resultative use among them, characterised as "to carry the action described by the base verb to a result":

i. intensifying the action of the imperfective verb rastolstet'

to grow stout, fat // grow stouter until one becomes stout raskormit'

to fatten // feed until one becomes large

\footnotetext{
${ }^{10}$ Only the perfective forms are listed; the secondary imperfective forms built from the prefixed verbs are ignored.
} 
ii. deriving verbs meaning 'with the help of the action of the base verb to perceive, take account of or explain in detail rassmotret' // be looking until perceived in detail to look at untill one discerns everything

iii. to carry out the action of the base verb to a result

\section{razbudit'}

to wake up // be waking up until one is awake

\section{rasserdit'}

to make angry // be making angry until one is angry

All these uses involve bounding states. All but the neutral resultative meaning are defined as external to the verb by selecting a dimension on which the bounding point is defined. The neutral resultative meaning refers to the dimension which is inherent in the semantics of the verb, since the becoming event of waking somebody is defined as getting more and more awake.

Formalising these observations, we assume that each perfective tag is associated with defaults which state what its bounding dimensions are, in a very general form. These may be further specified in the particular situation scheme. A perfective tag which has a neutral resultative meaning contains a default for $c_{-}$state. Additional restrictions as to which verb class is intended are possible, but skipped here. The dimensions particular to the perfective tag are also specified here. Thus, raz might have a requirement $\operatorname{dist}(x, e 2)$ stating that $x$ exists only in the distributed form in the c-state, for some states, reflecting Švedova's reading (i), cf. (69). Only one kind of bounding dimension is shown her, raz_b_dimension $(e 1, e 2)$. 


$$
\begin{aligned}
& A_{(\operatorname{ct}(R A Z))}= \\
& <\Gamma= \\
& \left\{\begin{array}{l}
\text { c_state_bound }(e 1, e 2) \& \text { c_state }(e 1, e 2) \rightarrow \text { raz_b_dimension }(e 1, e 2) \\
\text { dist_state_bound }(e 1, e 2) \& \sim c \_s t a t e \_b o u n d(e 1, e 2) \& c \_s t a t e(e 1, e 2) \\
\text { theme }(x, e 2) \& \text { dist }(x, e 2) \rightarrow \text { raz_b_dimension }(e 1, e 2) \\
\text { raz_b_dimension }(e 1, e 2) \rightarrow b \_d i m e n s i o n(e 1, e 2)
\end{array}\right\} \\
& \Delta=\left\{\begin{array}{l}
\text { c_state_bound }(e 1, e 2), \sim \text { state_bound }(e 1, e 2), \\
\text { dist_state_bound }(e 1, e 2), \sim \text { dist_state_bound }(e 1, e 2)
\end{array}\right\} \\
& \Pi=\emptyset \\
& C=\left\{\begin{array}{l}
\text { c_state_bound }(e 1, e 2) \& \sim c_{\_} \text {state_bound }(e 1, e 2) \rightarrow \perp \\
\text { dist_state_bound }(e 1, e 2) \& \sim \text { dist_state_bound }(e 1, e 2) \rightarrow \perp
\end{array}\right\}> \\
& \text { explain : }
\end{aligned}
$$

Different perfective tags are used to express neutral results in Russian, and there is no single prefix covering most of the verbs. However, there is some perfective tag practically for each verb which expresses its c-statebased neutral result. The default for $c_{-}$state $(e 1, e 2)$ is $c_{-} s t a t e \_b o u n d(e 1, e 2)$ Any other rule which uses $c_{-}$state $(e 1, e 2)$ must be conditioned on the conjugate default, $\sim c_{\_}$state_bound $(e 1, e 2)$.

\subsubsection{Russian perfective verbs are [+bounded]}

Before we go on, one comment is in order. It seems rather plausible to consider Russian perfective verbs as lexical counterparts to the English perfect. This is not always possible. In terms of the paper Russian perfective verbs are $[+\mathrm{b}($ ound $)]$, but not $[+\mathrm{r}-$ state $]$. In other words, the consstate in English is reserved for r-states. In Russian it is a bounding state. If a r-state in English involves a c-state, the English perfect can have a nice correspondence in Russian. Yet there are also regular differences between English perfect and perfective verbs in Russian.

- The first difference has been discussed already. R-states differ from c-states in that c-states may be transitory, r-states are permanent. He has baked a cake implies that the cake is there at the moment of utterance. The Russian perfective counterpart On vypek pirog has no such implication. The perfective form vypek of imperfective pek (was 
baking/baked) does not guarantee that the cake was not eaten in the past already, only that the c-state was reached somewhere in the past.

- Since Russian perfective verbs are never used in the present tense, a possible alternative could have been to use imperfective verbs, but they usually denote a process in the present tense, hence do not carry an implication that the c-state holds, cf. On pečet/vypekajet pirog (primary/secondary imperfective form)

- Curiously, in case of static verbs like live or trust the regular translation of the present perfect form is simple present of an imperfective verb, with the same implication of a state continuing till the moment of utterance. $(70,71)$.

(70) a. John has lived here for 20 years

b. Džon živet zdes' 20 let

Džon vs'u žizn' verit bratu
John all life trust-pres,imperf his brother
John has trusted his brother all his life

A perfective form, e. g. pro-žil zdes' 20 let has again no implication that the end of the 20 year period is at the moment of utterance.

- R-states are defined in English for processes which are in the past. In Russian their possible perfective equivalents are sometimes not defined: he has run has no precise context-equivalent translation in Russian. If the implicatures of the r-state are defined in a context, an adequate translation may be provided, however.

We therefore consider Russian perfective verbs to manifest the operation of event bounding, referring to bounding states and not to r-states as in the case of English perfect. However, the implications of the two may often, though not always, coincide ${ }^{11}$.

\footnotetext{
${ }^{11}$ I am aware that I am encroaching on the territory of aspect theory. Since a correct theory of Russian aspect is not the primary aim of the paper I do not want to get involved in disputes in this field. I only note for the purpose of orientation that the ideas expressed here are compatible with proposals of Maslov (Maslov, 1984) and Bulygina and Šmelev (Bulygina and Šmelev, 1997) on the nature of Russian aspect.
} 


\subsubsection{Absence of RC in Russian: An example of blocking}

Now let us return to the main hypothesis which will set the context of the explanation. Russian grammaticalises the relation of boundedness. To explain grammaticalization in the framework of the paper we postulate a grammatical feature $[+\mathrm{b}]$ with the following properties:

- it occurs on the V-prefix position, e. g. (72).

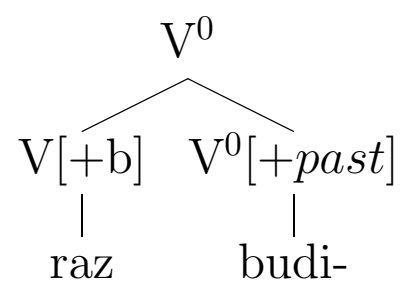

- it thus defines a syntactic relation which we will call $g f_{-} V_{-} a d j_{-} b$ for mnemonic reasons. It is a kind of lexical syntactic relation involving a verb.

- the syntactic relation $g f_{-} V_{-} a d j_{-} b$ is uniquely associated with the argument relation bounds. This relation has to be realized, if derivable, since it is grammaticalized. It is realized by a perfective tag.

- the relation bounds must be explained in any context, since it is grammaticalized by $[+b]$.

- in Russian bounds is mapped to the consstate argument relation. In other words, bounds is simultaneously an explanation of the consequent state in the structure of the situation.

- the relation bounds is explainable by the operation of event bounding.

- The particular properties of the class of event bounding are listed in the context of the perfective tag

To put it concisely, in Russian we have a context associated with the feature $[+b]$, with at least the following partial definition, cf. (73). 


$$
\begin{aligned}
& A_{(c t(r a z))}=<\Gamma=\left\{\begin{array}{l}
g f \_V \_a d j \_b((e 1, e 2)) \\
b \_d i m e n s i o n(e 1, e 2) \rightarrow \operatorname{bounds}(e 1, e 1) \\
c \_s t a t e(e 1, e 2) \rightarrow b \_\operatorname{dimension}(e 1, e 2)
\end{array}\right\} \\
& \Delta=\emptyset \\
& \Pi=\emptyset \\
& C=\emptyset> \\
& \text { explain : bounds }(e 1, e 2)
\end{aligned}
$$

Whenever a c-state is asserted to hold within the situation, it is considered to be a bounding dimension and automatically mapped to b_dimension. Other bounding dimensions are possible, and their general properties may be listed here, too.

The effect of blocking is now reconstructed in the following way. Since $[+\mathrm{b}]$ grammaticalises bounds $(e 1, e 2)$, this predicate has to be explained in any context. It can be explained, if b_dimension can be explained. The latter can be explained, if it can be explained as some bounding dimension with the help of a perfective tag. Since English uses c_state in RSP, only defaults for this predicate matter. Any Russian verb which has a natural c-state would create an alternative: either use some perfective tag which expresses its c-state, or use the English kind of defaults in (66), repeated here with their conjugates.

$$
\begin{aligned}
& A_{\left(c t\left(\oplus c_{-} \text {state }\right)\right)}= \\
& <\Gamma=\left\{\begin{array}{l}
\left.c t(s): \text { makecstate } 1\left(e, e_{b}, e_{r e s}\right), e_{\oplus}\right) \& \operatorname{cstate}\left(e, e_{b}\right) \\
\& e_{b} \leq e_{\text {res }} \& e_{\oplus}=e \oplus e_{\text {res }} \\
\rightarrow c \_ \text {state }\left(e, e_{r e s}\right) \\
\left.c t(s): \text { makecstate } 2\left(e, e_{b}, e_{r e s}\right), e_{\oplus}\right) \& \operatorname{cstate}\left(e, e_{b}\right) \\
\& c_{\_} \text {state }\left(e, e_{r e s}\right) \& e_{b} \leq e_{r e s} \\
\rightarrow e_{\text {res }} \leq e_{b}
\end{array}\right\} \\
& \Delta=\left\{\begin{array}{l}
\text { makecstate } 1\left(e, e_{b}, e_{r e s}, e_{\oplus}\right), \sim \text { makecstate } 1\left(e, e_{b}, e_{r e s}, e_{\oplus}\right) \\
\text { makecstate } 2\left(e, e_{b}, e_{r e s}, e_{\oplus}\right), \sim \text { makecstate } 2\left(e, e_{b}, e_{r e s}, e_{\oplus}\right)
\end{array}\right\} \\
& \Pi=\emptyset \\
& C=\left\{\begin{array}{l}
\text { makecstate } 1\left(e, e_{b}, e_{r e s}, e_{\oplus}\right) \& \sim \text { makecstate } 1\left(e, e_{b}, e_{r e s}, e_{\oplus}\right) \rightarrow \perp \\
\text { makecstate } 2\left(e, e_{b}, e_{r e s}, e_{\oplus}\right) \& \sim \text { makecstate } 2\left(e, e_{b}, e_{r e s}, e_{\oplus}\right) \rightarrow \perp
\end{array}\right\}> \\
& \text { explain: }
\end{aligned}
$$


Since these are mapping rule defaults, they must have conjugates. It is inessential whether these defaults are conditioned on the conjugate of the perfective tag, e. g. $\sim c_{-} s t a t e \_b o u n d(e 1, e 2)$ in the case of raz, or the defaults of the perfective tag are conditioned on the conjugates of the English defaults: one of them is excluded, if the other is applied. At this point we should consider the two perspectives of Gricean contexts. Let us begin with the hearer perspective.

Hearer perspective:

Learning Russian, a hearer has to learn how to interpret perfective tags. In particular, s/he has to learn that the operation of bounding in Russian introduces a consequent state in the situation, i. e. consequent states in Russian are explained by boundedness and boundedness is grammaticalized via $[+b]$. Since it is grammaticalized, it must be interpreted, i. e. explained. The only explanation available in Russian is via some perfective tag, since the bounding operation in Russian is split into different particular cases associated with different perfective tags and different verb classes.

Consider what happens when a hearer is confronted with a secondary predicate in a case in which the c-state is asserted to hold in the situation. $\mathrm{S} /$ he must assume that there is a c-state in the situation scheme which is the consequent state of the situation. Moreover, it has to be co-identified with the resultative state of the RSP. Adopting the co-identification defaults blocks the defaults associated with the neutral perfective tag. This, in its turn, prevents bounded from being explained. But the requirement is that it must be explained. So the perfective tag defaults cannot be dropped. Thus, the defaults of the co-identification context will be blocked.

Speaker perspective:

The speaker wants to communicate that an event is bounded by a c-state. In principle both the way via a perfective tag and the way via a secondary predicate are possible, since the speaker is not obliged to introduce $[+b]$. However, the speaker has to consider the hearer's perspective, too. And this settles the choice of $[+b]$ and some perfective tag.

Note that this account presuppose that, though both alternatives are viable at the time where the learning of the language begins, only one survives when the language has been learned. 


\subsection{Russian verb prefixes, prepositional phrases and the bounding operation}

Let us briefly return to the Russian translation J.K Jerome's text in (1.1). We mentioned two prefixes there. The prefix raz-, which was used with the verb pl'uščit' (flatten), provides here a good illustration of a c-state. To flatten is to make something flat, with flatness defined relative to the verb or a verb class. But the translation also contains a prepositional phrase $v$ lepešku glossed over as into a flat cake. The new form is obviously a result of the flattening. Is that not the case of resultative predication which was argued to be blocked in Russian?

In general, this seems to be the case of a prepositional RC. Such a construction is claimed to exist in English, so let us first consider it there. Though its scope is not quite clear, it seems to be based on t-states. Recall that the main difference between a t-state and a c-state was that a t-state may be specified extrinsically. Thus, running may have a t-state of running until one comes into the wood or until one gets tired. English uses a PP for the first case and a reflexive $\mathrm{RC}$ for the second, i. e. John ran into the wood and John ran himself tired. Russian also uses t-states. But nether c-states nor t-states must to be associated with boundedness, if they are not claimed to hold in the situation. Similarly, since nether RC based on adjectives, nor RC based on PPs in English are associated with grammaticalized boundedness. It is perfectly possible to have John was running into the wood and John was running himself tired. There is no resultative implicature for the progressive RC in English.

Prepositional phrases do not require co-identification of the c-state of the matrix event with the event of the secondary predicate. Probably they are not secondary predicates at all. What they do is simply define a t-state for the matrix event, enforcing it to have a culmination.

To return to the Russian case: the moment a t-state is claimed to hold in the situation, i. e. to be a consequent state, a perfective tag has to be used, since a t-state which holds in a situation is a bounding state, cf. $(75,76)$.

(75) Pet'a bežal v les

Peter run-imp-past in(to) wood

Peter was running into the wood 
Here, (75) does not imply that he was in the wood in the situation, (76) does exactly this. To accommodate t-states we should extend the definition of the bounding operation, but otherwise the explanation applies.

The second perfectivising prefix used in the translation, pro-, is an illustration of the complex nature of event bounding operation in Russian. Were we to describe the particular impact of the prefix pro- on the meaning of the verb, the description would run like this: penetrate the surface of an object by acting on the object in the way described by the verb. If a verb specifies an action which can be a cause of penetration and specifies the form of the penetration, the form is realized as an object and the object acted upon becomes a prepositional object, e. g. (77).

(77) pro- burit' skvažinu (v zemle)

(through-) drill a well in earth

to drill a well (in the earth)

Obviously, the c-state is characterised by the newly created object which is an aperture. However, there is also a t-state which locates the aperture in the object acted upon. Both of these states are results of the action, but one of them is expressed by the perfective tag, the other by a prepositional phrase. This is the case of a prefix adding some meaning. As a rule such meanings are c-states and dimensions for c-states.

The simplex verb bit' from the translation is a good illustration of this process. It does not allow the object which denote an aperture. It rather requires the object which receives the blows. Holes are bad as such objects. But beating may cause apertures, so when the perfective tag prois applied, it actually adds its own object which provides a c-state.

\section{A summary and some consequences}

The paper showed how some variation in the overt realisation of a subset of consequent states in Russian and English may be explained in an inferential theory of meaning by an appeal to structuring principles on inferences. 
The explanation thus contrasts with a very common tendency to explain this phenomenon in terms of the absence of some property of language in Russian, e. g. some syntactic feature, some syntactic or some lexical operation.

The theory is based on the assumption that the semantic interpretation of syntactic structures and the generation of syntactic structures of the language from communicative sense are inferential activities which take place in Gricean contexts. Gricean contexts are described as default rule systems with conjugate defaults (a notion similar to disjunctive rule ordering). Default rule systems in Gricean contexts are constructed and used under conditions optimising communication, as noted by Grice (Grice, 1989). One general construction principle is that two alternative interpretations which both have chances to be used, must block each other's application in the same context. If one is grammaticalsed and the other is not, the first one may completely block the other in all contexts.

The paper formalised a small inferential theory of interpretation. The inferential part was based on abductive reasoning in Poole systems. The semantics for RC in English proposed by Parsons and Rothstein was reworked in the abductive interpretation theory. The notions that lead to conflicting interpretations in Russian, provided an English-kind of rule is used there, were analysed. Their place in the interpretation theory was determined, using a proposal by Levin and Rappaport Hovav.

It was suggested that Russian has a grammaticalized event bounding operation, which English does not have. This operation forms a part of the rules mapping situation schemes to syntactic structures, i. e. part of the rule system called linking rules. The grammatical feature associated with this operation has to be interpreted, and gets interpreted via verb prfectivisation in Russian. Using the English-style RC in perfective contexts is impossible, because the necessity of interpretation of $[+b]$ is violated. The imperfective verb contexts in Russian cannot have the same interpretation, since they do not make claims about consequent states holding in the situation referred to by the sentence uttered ${ }^{12}$. The net result of this

\footnotetext{
${ }^{12}$ Imperfective verbs, especially with plural objects, may sometimes have either purely distributive or habitual perfective reading, similarly to simple past uses like While he wrote I read or He wrote a letter every week. The general property of Russian imperfective aspect is that it can be used for homogeneous events. Such homogeneous events may be built up from bounded atomic events, expressing iteration.
} 
situation is that the RC-type of interpretation is blocked to accommodate the bonding operation.

In general, the paper lends support to the position that the absence of $\mathrm{RC}$ in Russian is not due to the absence of a lexical operation or some syntactic feature there, but a consequence of a complex interplay of a number of factors which are operative in English, too. One prominent factor is that Russian grammaticalises the event bounding operation.

The most important implication of this paper is that general principles of an inferential syntax-semantics mapping might be responsible for some typological variation, eliminating this region from the typological parametric variation.

\section{Author's address}

Anatoli Strigin

ZAS Berlin

Jaegerstrasse 10-11

10117 Berlin

Germany

e-mail: strigin@zas.gwz-berlin.de

http://www.zas.gwz-berlin.de/mitarb/homepage/strigin/

\section{Acknowledgements}

I would like to thank Assinja Demjjanow, Hans Martin Gaertner, Manfred Krifka and Barbara Partee for their help. The usual disclaimers apply.

The difference to the English progressive is that the use of the Russian imperfective is not limited to homogeneous events. See Forsyth (1970) 


\section{References}

Bowers, J. (1993). The syntax of predication, Linguistic Inquiry 24: 591656.

Bréal, M. (1887). Essay de Sémantique. Quoted after the English translation Semantics: Studies in the Science of Meaning, Dover, New York, 1964.

Bulygina, T. V. and Šmelev, A. D. (1997). Jazykovaja konceptualizacija mira (na materiale russkogo jazyka). Pragmatika - Semantika - Lexikografija - Vid - Vremja - Lico - Modal'nost., Škola, Moskva.

Charniak, E. and McDermott, D. (1985). Introduction to Artifical Intelligence, Addison-Wesley, Reading, Massachusetts.

Demjjanow, A. and Strigin, A. (2001). Measure instrumental in russian, in G. Jäger, A. Strigin, C. Wilder and N. Zhang (eds), Papers on Predicative Constructions, Vol. 22 of ZAS Papers in Linguistics, Zentrum für Allgemeine Sprachwissenschaft, Sprachtypologie und Universalienforschung, Berlin, pp. 69-97.

Demjjanow, A. and Strigin, A. (2003). Real adjuncts in instrumental in Russian, in E. Lang, C. Maienborn and C. Fabricius-Hansen (eds), Modifying Adjuncts, Mouton de Gruyter, Berlin.

Eshghi, K. (1993). A tractable class of abduction problems, Proceedings of IJCAI-93, Chambérry.

Forsyth, J. (1970). A grammar of aspect. Usage and meaning in the Russian verb, Cambridge University Press, Cambridge.

Genesereth, M. R. and Nilsson, N. J. (1987). Logical Foundations of Artificial Intelligence, Morgan Kaufmann, Palo Alto.

Green, G. M. (1972). Some observations on the syntax and semantics of instrumetnal verbs, Papers from the eighth regional meeting of the Chicago Linguistic Society, Chicago Linguistic Society, Chicago, Illinois, pp. 83-97. 
Grice, H. P. (1975). Logic and conversation, in P. Cole and J. Morgan (eds), Syntax anbd Semantics, vol 3: Speech acts, Academic Press, New York, pp. 41-58. Reprinted in Grice (1989).

Grice, H. P. (1989). Studies in the Way of Words, Harward University Press, Cambridge, Massachusetts.

Hobbs, J., Stickel, M., Appelt, D. and Martin, P. (1993). Interpretation as abduction, Artificial Intelligence 63: 69-142.

Jespersen, O. (1924). The Philosophy of Grammar, Allen and Unwin, London.

Kamp, H. and Reyle, U. (1993). From Discourse to Logic, Kluwer, Dordrecht.

Kaufmann, I. (1995). O- and D-predicates: A semantic approach to the unaccusative-unergative distinction, Journal of Semantics 12: 377-427.

Kautz, H., Kearns, M. and Selman, B. (1995). Horn approximations of empirical data, Artificial Intelligence 74. 129-145.

Konolige, K. (1996). Abductive theories in artificial intelligence, in G. Brewka (ed.), Principles of Knowledge Representation, CSLI Publications, Stanford, pp. 129-152.

Krifka, M. (1992). Thematic relations as links between nominal reference and temporal constitution, in I. A. Sag and A. Szabolcsi (eds), Lexical Matters, CSLI, pp. 29-53.

Krifka, M. (1998). The origins of telicity, in S. Rothstein (ed.), Events and Grammar, Kluwer, pp. 197-235.

Laserson, P. (1992). Generalized conjunction and temporal modification, Linguistics and Philosophy 15: 381-410.

Legendre, G. (1997). Secondary predication and functional projections in french, Natural Language and Linguistic Theory 15: 42-87.

Levin, B. and Rappaport Hovav, M. (1995). Unaccusativity. At the SyntaxLexical Semantics Interface, The MIT Press, Cambridge, Mass. 
Levin, B. and Rappaport Hovav, M. (1999). Two structures for compositionally derived events, in T. Matthews and D. Strolovich (eds), Proceedings from SALT IX, Cornell University, Ithaca, pp. 199-223.

Lifschitz, V. (1996). Foundations of logic programming, in G. Brewka (ed.), Principles of Knowledge Representatino, CSLI Publications, Stanford, pp. $69-127$.

Maslov, J. S. (1984). Očerki po aspektologii, Izdatel'stvo Leningradskogo universiteta, chapter Vid i leksičeskoje značenije glagola v sovremennom russkom literaturnom jazyke, pp. 48-63. Reprint from Izvestija AN SSSR, otdel literatury i jazyka, tom 7 vypusk 4, 303-316, 1948.

McCarthy, J. and Buvač, S. (1998). Formalizing context (expanded notes), in A. Aliseda, R. van Glabbeek and D. Westerståhl (eds), Computing Natural Language, CSLI, Stanford, Cal., pp. 13-50.

McRoy, S. W. and Hirst, G. (1995). The repair of speech act misunderstanding by abductive inference, Computational Linguistics 18: 1-30.

Meyer-Klabunde, R. (1995). Processing connectives. A paper version of the talk at the DGFS session, Göttingen.

Moens, M. (1987). Tense, Aspect ans Temporal Reference, PhD thesis, Centre for Cognitive Science, University of Edinburgh.

Muggleton, S. (1996). Learning from positive data, Proceedings of the Sixth Inductive Logic Programming Workshop, pp. 225-244.

Nilsson, U. and Maluszyński (1990). Logic, Programming and Prolog, Wiley, Chichester.

Norvig, P. and Wilensky, R. (1993). A critical evaluation of commensurable abduction models for semantic interpretation, in H. Karlgren (ed.), Proceedings of COLING-90, pp. 225-230.

Parsons, T. (1990). Events in the semantics of English. A study in subatomic semantics, The MIT Press, Cambridge, Mass.

Peirce, C. S. (1992). Reasoning and The Logic of Things, Harvard University Press, Cambridge, Mass. Edited by Kenneth Laine Ketner. 
Peškovskij, A. J. (1956). Russkij sintaksis v naučnom osveščenii, 7 edn, Učpedgiz, Moskow.

Poole, D. (1988a). A logical framework for default reasoning, Artificial Intelligence 36: 27-47.

Poole, D. (1988b). A logical framework for default reasoning, Artificial Intelligence 36: 27-47.

Rappaport Hovav, M. and Levin, B. (2001). An event structure account of English resultatives, Language 77(4): 766-797.

Reiter, R. (1987). Nonmonotonic reasoning, Annual Review of Computer Science 2: 147-186.

Rothstein, S. (2001). What are incremental themes?, in G. Jaeger, A. Strigin, C. Wilder and N. Zhang (eds), Papers on Predicative Constructions, Vol. 22, Zentrum fuer allgemeine Sprachwissenschaft, Sprachtypologie und Universalienforschung, Zentrum fuer allgemeine Sprachwissenschaft, Sprachtypologie und Universalienforschung, Berlin, pp. 139158. Proceedings of the Workshop on Secondary Predication, October 2000, Berlin.

Rothstein, S. (2003). Modifying Adjuncts, Mouton de Gruyter, Berlin, chapter Secondary Predication and Aspectual Structure, pp. 553-590.

Strigin, A. and Demjjanow, A. (2001). Secondary predication in Russian, ZAS Papers in Linguistics 25: 1-79.

Švedova, N. Y. (ed.) (1970). Grammatika sovremennogo russkogo literaturnogo jazyka, Nauka, Moscow.

Tenny, C. (1994). Aspectual Roles and the Syntax-Semantics Interface, Kluwer, Dordrecht.

Tenny, C. (1995). How motion verbs are special: The interaction of semantic and pragmatic information in aspectual verb meanings, Pragmatics and cognition 3(1): 31-73.

Thomson, A. J. and Martinet, A. V. (1960). A Practical English Grammar, Oxford Unversity Press. 
Vasishth, S. and Kruijiff, G.-J. M. (2001). Sentence Processing as Abduction+Deduction, Ohio State University Working Papers in Linguistics, Vol. 56, OSU Press, pp. 183-207.

Wade, T. (1992). A Comprehensive Russian Grammar, Blackwell, Oxford.

Winkler, S. (1997). Focus and Secondary Predication, Mouton de gruyter, Berlin.

Wunderlich, D. (1997). Argument extension by lexical adjunction, Journal of Semantics 14: 95-142. 